\title{
Morrey Sequence Spaces: Pitt's Theorem and Compact Embeddings
}

\author{
Dorothee D. Haroske ${ }^{1}$. Leszek Skrzypczak ${ }^{2}$
}

Received: 29 June 2018 / Revised: 11 December 2018 / Accepted: 2 January 2019 /

Published online: 28 March 2019

(c) The Author(s) 2019

\begin{abstract}
Morrey (function) spaces and, in particular, smoothness spaces of Besov-Morrey or Triebel-Lizorkin-Morrey type have enjoyed a lot of interest recently. Here we turn our attention to Morrey sequence spaces $m_{u, p}=m_{u, p}\left(\mathbb{Z}^{d}\right), 0<p \leq u<\infty$, which have yet been considered almost nowhere. They are defined as natural generalizations of the classical $\ell_{p}$ spaces. We consider some basic features, embedding properties, a predual, a corresponding version of Pitt's compactness theorem, and further characterize the compactness of embeddings of related finite-dimensional spaces.
\end{abstract}

Keywords Morrey sequence spaces · Pitt's theorem · compact embeddings · entropy numbers

Mathematics Subject Classification 46E35 - 46A45 - 46B45

\section{Introduction}

Morrey (function) spaces and, in particular, smoothness spaces of Besov-Morrey or Triebel-Lizorkin-Morrey type were studied in recent years quite intensively and systematically. Decomposition methods like atomic or wavelet characterizations require suitably adapted sequence spaces. This has been done to some extent already. We are

Communicated by Pencho Petrushev.

Leszek Skrzypczak was supported by National Science Center, Poland, Grant No. 2014/15/B/ST1/00164.

Leszek Skrzypczak

1skrzyp@amu.edu.pl

Dorothee D. Haroske

dorothee.haroske@uni-rostock.de

1 Institute of Mathematics, University of Rostock, 18057 Rostock, Germany

2 Faculty of Mathematics and Computer Science, Adam Mickiewicz University, ul. Umultowska 87, 61-614 Poznan, Poland 
interested in related sequence spaces of Morrey type, but first we briefly review some basic facts about the much more prominent function spaces of Morrey type.

Originally, Morrey spaces were introduced in [32], when studying solutions of second order quasi-linear elliptic equations in the framework of Lebesgue spaces. They can be understood as a complement (generalization) of the Lebesgue spaces $L_{p}\left(\mathbb{R}^{d}\right)$. In particular, the Morrey space $\mathcal{M}_{u, p}, 0<p \leq u<\infty$, is defined as the collection of all complex-valued Lebesgue measurable functions on $\mathbb{R}^{d}$ such that

$$
\left\|f \mid \mathcal{M}_{u, p}\left(\mathbb{R}^{d}\right)\right\|=\sup _{x \in \mathbb{R}^{d}, R>0} R^{d\left(\frac{1}{u}-\frac{1}{p}\right)}\left(\int_{B(x, R)}|f(y)|^{p} \mathrm{~d} y\right)^{\frac{1}{p}}<\infty
$$

where $B(x, R)=\left\{y \in \mathbb{R}^{d}:|x-y|<R\right\}$ are the usual balls centered at $x \in \mathbb{R}^{d}$ with radius $R>0$. Obviously, $\mathcal{M}_{p, p}\left(\mathbb{R}^{d}\right)=L_{p}\left(\mathbb{R}^{d}\right)$, and $\mathcal{M}_{u, p}\left(\mathbb{R}^{d}\right)=\{0\}$ if $p>u$. Moreover, $\mathcal{M}_{\infty, p}\left(\mathbb{R}^{d}\right)=L_{\infty}\left(\mathbb{R}^{d}\right)$ such that the usual assumption is $p \leq u<\infty$. As can be seen from the definition, Morrey spaces describe the local behavior of the $L_{p}$ norm, which makes them useful when describing the local behavior of solutions of nonlinear partial differential equations, cf. [22, 25-26, 27, 30, 31, 50]. Furthermore, applications in harmonic analysis and potential analysis can be found in the papers [2-4]. For more information, we refer to the books [1] and [48] and, in particular, to the fine surveys $[46,47]$ by Sickel.

As for the smoothness spaces of Morrey type, aside from Besov-Morrey spaces $\mathcal{N}_{u, p, q}^{s}\left(\mathbb{R}^{d}\right)$ in $[22,30,31]$, and their counterparts Triebel-Lizorkin-Morrey spaces $\mathcal{E}_{u, p, q}^{s}\left(\mathbb{R}^{d}\right), \mathrm{cf}$. [49], their atomic and wavelet characterizations were already described in the papers $[39,41-44]$, and we simplified the appearing sequence spaces $n_{u, p, q}^{s}$ in [18] to some extent. There are further related approaches to Besov-type spaces $B_{p, q}^{s, \tau}\left(\mathbb{R}^{d}\right)$ and Triebel-Lizorkin-type spaces $F_{p, q}^{s, \tau}\left(\mathbb{R}^{d}\right)$, cf. [48] with forerunners in $[12-14,55,56]$. Triebel provided a third approach, so-called local and hybrid spaces, in $[53,54]$, but they coincide with appropriately chosen spaces of type $B_{p, q}^{s, \tau}\left(\mathbb{R}^{d}\right)$ or $F_{p, q}^{s, \tau}\left(\mathbb{R}^{d}\right)$, cf. [57].

Recently, based on some discussion at the conference "Banach Spaces and Operator Theory with Applications" in Poznan in July 2017, we found that Morrey sequence spaces $m_{u, p}=m_{u, p}\left(\mathbb{Z}^{d}\right), 0<p \leq u<\infty$, have been considered almost nowhere. The paper [6] concerns another type of discretization than we have in mind. To the best of our knowledge, there is only the paper [16] (and an interesting application in [17]) so far that is devoted to this subject. They are defined as natural generalizations of $\ell_{p}=\ell_{p}\left(\mathbb{Z}^{d}\right)$ via

$$
\begin{aligned}
m_{u, p}= & \left\{\lambda=\left\{\lambda_{k}\right\}_{k \in \mathbb{Z}^{d}} \subset \mathbb{C}:\right. \\
& \left\|\left.\lambda\left|m_{u, p} \|=\sup _{j \in \mathbb{N}_{0} ; m \in \mathbb{Z}^{d}}\right| Q_{-j, m}\right|^{\frac{1}{u}-\frac{1}{p}}\left(\sum_{k: Q_{0, k} \subset Q_{-j, m}}\left|\lambda_{k}\right|^{p}\right)^{\frac{1}{p}}<\infty\right\},
\end{aligned}
$$


where $Q_{j, m}=2^{-j}\left(m+[0,1)^{d}\right)$ are dyadic cubes of side length $2^{-j}, j \in \mathbb{Z}, m \in \mathbb{Z}^{d}$. Clearly, $m_{p, p}=\ell_{p}$.

We consider some basic features in Sect. 2 and present our main embedding result, Theorem 3.1, in Sect. 3, which reads as follows: Let $0<p_{1} \leq u_{1}<\infty$ and $0<$ $p_{2} \leq u_{2}<\infty$. Then the embedding

$$
m_{u_{1}, p_{1}} \hookrightarrow m_{u_{2}, p_{2}}
$$

is continuous if, and only if, the following conditions hold:

$$
u_{1} \leq u_{2} \quad \text { and } \quad \frac{p_{2}}{u_{2}} \leq \frac{p_{1}}{u_{1}}
$$

The embedding is never compact.

In Sect. 4, we describe a pre-dual $\mathcal{X}_{u, p}$ of $m_{u, p}, 1 \leq p<u<\infty$, which is a separable Banach space, unlike $m_{u, p}$.

Dealing with the closure $m_{u, p}^{00}$ of finite sequences in $m_{u, p}$, we obtain a counterpart to Pitt's theorem [37] in our setting as follows, see Theorem 5.3 below: Let $1<p<$ $u<\infty$ and $1 \leq q<\infty$. Then any bounded linear operator

$$
T: m_{u, p}^{00} \rightarrow \ell_{q}
$$

is compact. The above sequence spaces are not rearrangement invariant. Further information about the Pitt theorem in rearrangement invariant setting can be found in [8] and [28].

Finally, we further characterize the compactness of embeddings of related finitedimensional spaces and obtain for the asymptotic behavior of the dyadic entropy numbers of such a finite-dimensional embedding that

$$
e_{k}\left(\mathrm{id}_{j}: m_{u_{1}, p_{1}}^{2^{j d}} \rightarrow m_{u_{2}, p_{2}}^{2^{j d}}\right) \sim 2^{-k 2^{-j d}} 2^{j d\left(\frac{1}{u_{2}}-\frac{1}{u_{1}}\right)}
$$

where $j \in \mathbb{N}, 0<p_{i} \leq u_{i}<\infty, i=1,2$, and $k \in \mathbb{N}_{0}$ with $k \gtrsim 2^{j d}$.

\section{Morrey Sequence Spaces}

\subsection{Preliminaries}

First we fix some notation. By $\mathbb{N}$ we denote the set of natural numbers, by $\mathbb{N}_{0}$ the set $\mathbb{N} \cup\{0\}$, and by $\mathbb{Z}^{d}$ the set of all lattice points in $\mathbb{R}^{d}$ having integer components. For $a \in \mathbb{R}$, let $\lfloor a\rfloor:=\max \{k \in \mathbb{Z}: k \leq a\}$ and $a_{+}:=\max \{a, 0\}$. All unimportant positive constants will be denoted by $C$, occasionally with subscripts. By the notation $A \lesssim B$, we mean that there exists a positive constant $C$ such that $A \leq C B$, whereas the symbol $A \sim B$ stands for $A \lesssim B \lesssim A$. We denote by $|\cdot|$ the Lebesgue measure when applied to measurable subsets of $\mathbb{R}^{d}$. 
Given two (quasi-) Banach spaces $X$ and $Y$, we write $X \hookrightarrow Y$ if $X \subset Y$ and the natural embedding of $X$ into $Y$ is continuous.

For $0<p<\infty$, we denote by $\ell_{p}=\ell_{p}\left(\mathbb{Z}^{d}\right)$,

$$
\ell_{p}\left(\mathbb{Z}^{d}\right)=\left\{\lambda=\left\{\lambda_{k}\right\}_{k \in \mathbb{Z}^{d}} \subset \mathbb{C}:\left\|\lambda \mid \ell_{p}\right\|=\left(\sum_{k \in \mathbb{Z}^{d}}\left|\lambda_{k}\right|^{p}\right)^{\frac{1}{p}}<\infty\right\},
$$

complemented by

$$
\ell_{\infty}\left(\mathbb{Z}^{d}\right)=\left\{\lambda=\left\{\lambda_{k}\right\}_{k \in \mathbb{Z}^{d}} \subset \mathbb{C}:\left\|\lambda\left|\ell_{\infty} \|=\sup _{k \in \mathbb{Z}^{d}}\right| \lambda_{k} \mid<\infty\right\} .\right.
$$

If $\left\{\lambda_{\nu}^{*}\right\}_{\nu \in \mathbb{N}}$ stands for a nonincreasing rearrangement of a sequence $\lambda=\left\{\lambda_{k}\right\}_{k \in \mathbb{Z}^{d}} \in$ $\ell_{u}\left(\mathbb{Z}^{d}\right), 0<u<\infty$, then

$$
\ell_{u, \infty}\left(\mathbb{Z}^{d}\right)=\left\{\lambda=\left\{\lambda_{k}\right\}_{k \in \mathbb{Z}^{d}} \subset \mathbb{C}:\left\|\lambda \mid \ell_{u, \infty}\right\|=\sup _{v \in \mathbb{N}} v^{1 / u} \lambda_{v}^{*}<\infty\right\}
$$

denote the Lorentz sequence spaces, as usual. Finally, we adopt the custom to denote by $c=c\left(\mathbb{Z}^{d}\right), c_{0}=c_{0}\left(\mathbb{Z}^{d}\right)$, and $c_{00}=c_{00}\left(\mathbb{Z}^{d}\right)$ the corresponding subspaces of $\ell_{\infty}\left(\mathbb{Z}^{d}\right)$ of convergent, null, and finite sequences, respectively; that is,

$$
\begin{aligned}
c & =\left\{\lambda=\left\{\lambda_{k}\right\}_{k \in \mathbb{Z}^{d}} \in \ell_{\infty}: \exists \mu \in \mathbb{C}:\left|\lambda_{k}-\mu\right| \underset{|k| \rightarrow \infty}{\longrightarrow} 0\right\}, \\
c_{0} & =\left\{\lambda=\left\{\lambda_{k}\right\}_{k \in \mathbb{Z}^{d}} \in \ell_{\infty}:\left|\lambda_{k}\right| \underset{|k| \rightarrow \infty}{\longrightarrow} 0\right\}, \\
c_{00} & =\left\{\lambda=\left\{\lambda_{k}\right\}_{k \in \mathbb{Z}^{d}} \in \ell_{\infty}: \exists r_{0} \in \mathbb{N}_{0}: \lambda_{k}=0 \text { for }|k|>r_{0}\right\} .
\end{aligned}
$$

As we mostly deal with sequence spaces on $\mathbb{Z}^{d}$, we shall often omit it from their notation, for convenience.

\subsection{The Concept}

Let $Q_{j, m}, j \in \mathbb{Z}, m \in \mathbb{Z}^{d}$, denote the usual dyadic cubes in $\mathbb{R}^{d}$, i.e., $Q_{0,0}=[0,1)^{d}$ and $Q_{j, m}=2^{-j}\left(m+Q_{0,0}\right)$.

Definition 2.1 Let $0<p \leq u<\infty$. We define $m_{u, p}=m_{u, p}\left(\mathbb{Z}^{d}\right)$ by

$$
\begin{aligned}
m_{u, p}\left(\mathbb{Z}^{d}\right)= & \left\{\lambda=\left\{\lambda_{k}\right\}_{k \in \mathbb{Z}^{d}} \subset \mathbb{C}:\right. \\
& \left\|\left.\lambda\left|m_{u, p} \|=\sup _{j \in \mathbb{N}_{0} ; m \in \mathbb{Z}^{d}}\right| Q_{-j, m}\right|^{\frac{1}{u}-\frac{1}{p}}\left(\sum_{k: Q_{0, k} \subset Q_{-j, m}}\left|\lambda_{k}\right|^{p}\right)^{\frac{1}{p}}<\infty\right\} .
\end{aligned}
$$


Remark 2.2 In [16] the corresponding one-dimensional counterpart was introduced and studied.

Proposition 2.3 Let $0<p \leq u<\infty$.

(i) $m_{u, p}$ is a (quasi-) Banach space.

(ii) If $u=p$, then $m_{u, u}=\ell_{u}$; if $u<p$, then $m_{u, p}=\{0\}$. If $p_{1} \leq p_{2} \leq u$, then $m_{u, p_{2}} \hookrightarrow m_{u, p_{1}}$.

(iii) For any $p$ and $u$, we have $m_{u, p} \hookrightarrow \ell_{\infty}$.

(iv) If $p<u$, then $\ell_{u, \infty} \hookrightarrow m_{u, p}$.

(v) If $p<u$, then $m_{u, p}$ and $c_{0}$, c are incomparable; that is, $m_{u, p} \not \subset c_{0}, m_{u, p} \not \subset c$, $c_{0} \not \subset m_{u, p}, c \not \subset m_{u, p}$.

Proof Part (i) is standard; the completeness can be shown similarly to the (onedimensional) counterpart in [16].

The first two assertions in (ii) are obvious; the monotonicity in $p$ is a matter of Hölder's inequality. Concerning (iii), clearly for any $m \in \mathbb{Z}^{d}$,

$$
\begin{aligned}
& \left|\lambda_{m}\right|=\left|Q_{0, m}\right|^{\frac{1}{u}-\frac{1}{p}}\left(\sum_{k: Q_{0, k} \subset Q_{0, m}}\left|\lambda_{k}\right|^{p}\right)^{\frac{1}{p}} \\
& \leq \sup _{j \in \mathbb{N}_{0} ; m \in \mathbb{Z}^{d}}\left|Q_{-j, m}\right|^{\frac{1}{u}-\frac{1}{p}}\left(\sum_{k: Q_{0, k} \subset Q_{-j, m}}\left|\lambda_{k}\right|^{p}\right)^{\frac{1}{p}}=\left\|\lambda \mid m_{u, p}\right\|,
\end{aligned}
$$

such that finally, taking the supremum over all $m \in \mathbb{Z}^{d}$,

$$
\left\|\lambda\left|\ell_{\infty}\|\leq\| \lambda\right| m_{u, p}\right\|
$$

We prove (iv). Let $\left\{\lambda_{\nu}^{*}\right\}_{v \in \mathbb{N}}$ be a nonincreasing rearrangement of a sequence $\lambda=$ $\left\{\lambda_{k}\right\}_{k \in \mathbb{Z}^{d}}$. Then for any cube $Q_{-j, m}$, we have

$$
\begin{aligned}
\left(\sum_{k: Q_{0, k} \subset Q_{-j, m}}\left|\lambda_{k}\right|^{p}\right)^{1 / p} & \leq\left(\sum_{\nu=1}^{2^{j d}}\left|\lambda_{v}^{*}\right|^{p}\right)^{1 / p} \leq \sup _{r \in \mathbb{N}} r^{1 / u} \lambda_{r}^{*}\left(\sum_{\nu=1}^{2^{j d}} v^{-p / u}\right)^{1 / p} \\
& \leq C\left\|\left.\lambda\left|\ell_{u, \infty} \|\right| Q_{-j, m}\right|^{\frac{1}{p}-\frac{1}{u}}\right.
\end{aligned}
$$

since $p<u$ and $\left|Q_{-j, m}\right|=2^{j d}$. Hence $\left\|\lambda\left|m_{u, p}\|\leq C\| \lambda\right| \ell_{u, \infty}\right\|$.

It remains to deal with (v). Consider first the constant sequence $\lambda^{1}=\{1\}_{k \in \mathbb{Z}^{d}} \in c$. Then

$$
\left\|\left.\lambda^{1}\left|m_{u, p} \|=\sup _{j \in \mathbb{N}_{0} ; m \in \mathbb{Z}^{d}}\right| Q_{-j, m}\right|^{\frac{1}{u}-\frac{1}{p}}\left|Q_{-j, m}\right|^{\frac{1}{p}}=\sup _{j \in \mathbb{N}_{0}} 2^{j d / u}=\infty,\right.
$$

which disproves $c \subset m_{u, p}$ (and simultaneously strengthens (iii) by $m_{u, p} \subsetneq \ell_{\infty}$ ). A slight modification disproves $c_{0} \subset m_{u, p}$ : choose $\varepsilon$ such that $0<\varepsilon<\frac{d}{u}$, and consider $\tilde{\lambda}=\left\{\tilde{\lambda}_{k}\right\}_{k \in \mathbb{Z}^{d}}$ given by $\tilde{\lambda}_{k}=|k|^{-\varepsilon}$. Then $\tilde{\lambda} \in c_{0}$. On the other hand, 


$$
\left\|\left.\tilde{\lambda}\left|m_{u, p} \| \geq c \sup _{j \in \mathbb{N}_{0}}\right| Q_{-j, 0}\right|^{\frac{1}{u}-\frac{1}{p}} 2^{-j \varepsilon}\left|Q_{-j, 0}\right|^{\frac{1}{p}}=c \sup _{j \in \mathbb{N}_{0}} 2^{j(d / u-\varepsilon)}=\infty,\right.
$$

which gives $c_{0} \not \subset m_{u, p}$.

Now consider a special sequence $\lambda=\left\{\lambda_{k}\right\}_{k \in \mathbb{Z}^{d}}$ that looks as follows:

$$
\lambda_{k}= \begin{cases}1 & \text { if } k=\left(2^{r}, 0, \ldots, 0\right) \text { for some } r \in \mathbb{N} \\ 0 & \text { otherwise }\end{cases}
$$

Obviously $\lambda \notin c$; in particular, $\lambda \notin c_{0}$. Now, by construction,

$\left\|\left.\lambda\left|m_{u, p} \| \leq c \sup _{j \in \mathbb{N}_{0}}\right| Q_{-j, 0}\right|^{\frac{1}{u}-\frac{1}{p}}\left(\sum_{k: Q_{0, k} \subset Q_{-j, 0}}\left|\lambda_{k}\right|^{p}\right)^{\frac{1}{p}} \leq c^{\prime} \sup _{r \in \mathbb{N}} 2^{r d\left(\frac{1}{u}-\frac{1}{p}\right)} r^{\frac{1}{p}}<\infty\right.$.

So the subspaces $c_{0}, c$, and $m_{u, p}$ of $\ell_{\infty}$ are incomparable in the above sense.

Remark 2.4 Obviously, Definition 2.1 gives the discrete counterpart of $\mathcal{M}_{u, p}\left(\mathbb{R}^{d}\right)$ in view of (1.1). More precisely, given some sequence $\lambda=\left\{\lambda_{k}\right\}_{k \in \mathbb{Z}^{d}} \in m_{u, p}$,

$$
f_{\lambda}=\sum_{k \in \mathbb{Z}^{d}} \lambda_{k} \chi Q_{0, k} \in \mathcal{M}_{u, p}
$$

where $\chi_{A}$ denotes the characteristic function of a set $A \subset \mathbb{R}^{d}$, as usual.

Conversely, let $f_{0} \in \mathcal{M}_{u, p}$, and define $\lambda^{0}=\left\{\lambda_{k}^{0}\right\}_{k \in \mathbb{Z}^{d}}$ by $\lambda_{k}^{0}=\left(\int_{Q_{0, k}}\left|f_{0}(y)\right|^{p}\right.$ $\mathrm{d} y)^{1 / p}, k \in \mathbb{Z}^{d}$. Then $\left\|\lambda^{0}\left|m_{u, p}\|\leq\| f_{0}\right| \mathcal{M}_{u, p}\right\|$. Hence there is some obvious correspondence between the Morrey sequence and function spaces, respectively.

Remark 2.5 As in the case of the function spaces $\mathcal{M}_{u, p}\left(\mathbb{R}^{d}\right)$, one might complement Definition 2.1 in the case of $0<p \leq u=\infty$ by

$$
\begin{aligned}
m_{\infty, p}\left(\mathbb{Z}^{d}\right)= & \left\{\lambda=\left\{\lambda_{k}\right\}_{k \in \mathbb{Z}^{d}} \subset \mathbb{C}:\right. \\
& \left\|\left.\lambda\left|m_{\infty, p} \|=\sup _{j \in \mathbb{N}_{0} ; m \in \mathbb{Z}^{d}}\right| Q_{-j, m}\right|^{-\frac{1}{p}}\left(\sum_{k: Q_{0, k} \subset Q_{-j, m}}\left|\lambda_{k}\right|^{p}\right)^{\frac{1}{p}}<\infty\right\},
\end{aligned}
$$

where in the case of $p=u=\infty$, the latter sum has to be replaced by the supremum, as usual. Using this definition, we can show that $m_{\infty, p}=\ell_{\infty}$. This is obvious for $p=u=\infty$, so let us assume $p<u=\infty$. In view of Proposition 2.3(iii), it remains to show that $\ell_{\infty} \hookrightarrow m_{\infty, p}$ if $p<u=\infty$. Let $\lambda \in \ell_{\infty}$. Thus for any $j \in \mathbb{N}_{0}$ and $m \in \mathbb{Z}^{d}$, 


$$
\left|Q_{-j, m}\right|^{-\frac{1}{p}}\left(\sum_{k: Q_{0, k} \subset Q_{-j, m}}\left|\lambda_{k}\right|^{p}\right)^{\frac{1}{p}} \leq\left|Q_{-j, m}\right|^{-\frac{1}{p}}\left\|\lambda\left|\ell_{\infty}\left\|\left|Q_{-j, m}\right|^{\frac{1}{p}}=\right\| \lambda\right| \ell_{\infty}\right\|,
$$

which results in $\left\|\lambda\left|m_{\infty, p}\|\leq\| \lambda\right| \ell_{\infty}\right\|$.

Remark 2.6 Let $Q$ denote an arbitrary closed cube in $\mathbb{R}^{d}$ with $|Q| \geq 1$. We put

$$
\begin{aligned}
& \left\|\left.\lambda\left|m_{u, p} \|_{(1)}=\sup _{Q:|Q| \geq 1}\right| Q\right|^{\frac{1}{u}-\frac{1}{p}}\left(\sum_{k: Q_{0, k} \subset Q}\left|\lambda_{k}\right|^{p}\right)^{\frac{1}{p}},\right. \\
& \left\|\left.\lambda\left|m_{u, p} \|_{(2)}=\sup _{Q:|Q| \geq 1}\right| Q\right|^{\frac{1}{u}-\frac{1}{p}}\left(\sum_{k: Q_{0, k} \cap Q \neq \emptyset}\left|\lambda_{k}\right|^{p}\right)^{\frac{1}{p}} .\right.
\end{aligned}
$$

Then $\left\|\lambda \mid m_{u, p}\right\|_{(1)}$ and $\left\|\lambda \mid m_{u, p}\right\|_{(2)}$ are equivalent norms in $m_{u, p}$. It is obvious that $\left\|\lambda\left|m_{u, p}\|\leq\| \lambda\right| m_{u, p}\right\|_{(1)} \leq\left\|\lambda \mid m_{u, p}\right\|_{(2)}$. On the other hand, let $Q$ be a cube centered at $x_{0}$ with size $r \geq 1$. We take a cube $\widetilde{Q}$ centered at $x_{0}$ with size $r+2$. If we choose $j$ in such a way that $2^{j-1}<r+2 \leq 2^{j}$, then there are at most $2^{d}$ dyadic cubes $Q_{-j, m_{i}}$ such that $\bar{Q}_{-j, m_{i}}$ cover $\widetilde{Q}$. Then $\left|Q_{-j, m}\right| \leq 2^{d}|\widetilde{Q}| \leq 2^{2 d}|Q|$, and in consequence,

$$
\left\|\lambda\left|m_{u, p}\left\|_{(2)} \leq c_{d, u, p}\right\| \lambda\right| m_{u, p}\right\|_{(1)} \leq C_{d, u, p}\left\|\lambda \mid m_{u, p}\right\| .
$$

Proposition 2.7 Let $N \in \mathbb{N}, 0<p_{j} \leq u_{j}<\infty, j=1, \ldots, N$, and $\lambda^{(j)}=$ $\left\{\lambda_{k}^{(j)}\right\}_{k \in \mathbb{Z}^{d}} \in m_{u_{j}, p_{j}}, j=1, \ldots, N$. Then $\lambda^{(1)} \cdots \lambda^{(N)}=\left\{\lambda_{k}^{(1)} \cdots \lambda_{k}^{(N)}\right\}_{k \in \mathbb{Z}^{d}} \in m_{u, p}$, with

$$
\left\|\lambda^{(1)} \cdots \lambda^{(N)}\left|m_{u, p}\|\leq\| \lambda^{(1)}\right| m_{u_{1}, p_{1}}\right\| \cdots\left\|\lambda^{(N)} \mid m_{u_{N}, p_{N}}\right\|
$$

where

$$
\frac{1}{u}=\sum_{j=1}^{N} \frac{1}{u_{j}} \text { and } \frac{1}{p} \geq \sum_{j=1}^{N} \frac{1}{p_{j}} .
$$

Proof Obviously $0<\frac{1}{u}=\sum_{j=1}^{N} \frac{1}{u_{j}} \leq \sum_{j=1}^{N} \frac{1}{p_{j}} \leq \frac{1}{p}$ such that $m_{u, p}$ is well defined for $0<p \leq u<\infty$. The rest is iterated application of Hölder's inequality.

Proposition 2.8 Let $0<p \leq u<\infty, \lambda \in m_{u, p}$, and $0<r<\infty$. Then $|\lambda|^{r}=$ $\left\{\left|\lambda_{k}\right|^{r}\right\}_{k \in \mathbb{Z}^{d}} \in m_{u / r, p / r}$ with

$$
\left\||\lambda|^{r}\left|m_{\frac{u}{r}, \frac{p}{r}}\|=\| \lambda\right| m_{u, p}\right\|^{r}
$$

Proof This follows by the definition immediately. 
Proposition 2.9 Let $0<p<u \leq \infty$. Then $m_{u, p}$ is nonseparable.

Proof If $u=\infty$, then $m_{\infty, p}=\ell_{\infty}$ in view of Remark 2.6, and the result is well known. So assume $0<p<u<\infty$ now. Let $E$ be a subset of $\mathbb{N}$. We consider the following sequences $\lambda^{(E)}$ defined by

$$
\lambda_{k}^{(E)}= \begin{cases}1 & \text { if } \quad k=\left(2^{r}, 0, \ldots, 0\right) \text { and } r \in E \\ 0 & \text { otherwise }\end{cases}
$$

It should be clear that

$$
\left\|\lambda^{(E)}\left|m_{u, p}\|\leq\| \lambda^{(\mathbb{N})}\right| m_{u, p}\right\|
$$

So all the sequences belong to $m_{u, p}\left(\mathbb{Z}^{d}\right)$ if $\lambda^{(\mathbb{N})} \in m_{u, p}\left(\mathbb{Z}^{d}\right)$. For any cube $Q_{-j, m}$, we have by construction,

$$
\begin{aligned}
\left|Q_{-j, m}\right|^{\frac{1}{u}-\frac{1}{p}}\left(\sum_{k: Q_{0, k} \subset Q_{-j, m}}\left|\lambda_{k}^{(\mathbb{N})}\right|^{p}\right)^{\frac{1}{p}} & \leq\left|Q_{-j, 0}\right|^{\frac{1}{u}-\frac{1}{p}}\left(\sum_{k: Q_{0, k} \subset Q_{-j, 0}}\left|\lambda_{k}^{(\mathbb{N})}\right|^{p}\right)^{\frac{1}{p}} \\
& \leq 2^{j d\left(\frac{1}{u}-\frac{1}{p}\right)} j^{\frac{1}{p}} \leq C<\infty .
\end{aligned}
$$

Hence $\lambda^{(\mathbb{N})} \in m_{u, p}\left(\mathbb{Z}^{d}\right)$. If $E$ and $F$ are different subsets of $\mathbb{N}$, then

$$
\left\|\lambda^{(E)}-\lambda^{(F)} \mid m_{u, p}\right\| \geq 1 .
$$

Thus $m_{u, p}\left(\mathbb{Z}^{d}\right)$ contains a noncountable set of sequences such that the distance between two different elements of it is at least 1.

Remark 2.10 Let us mention briefly that in [16], further (one-dimensional) approaches to weak and generalized Morrey sequence spaces were considered.

\section{Embeddings}

We prove our main result about embeddings of different Morrey sequence spaces. Here we also use and adapt some ideas of our paper [18], cf. the proof of Theorem 3.2 there. A similar construction was used by P. Olsen in [33], cf. the proof of Theorem 10 in [33].

Theorem 3.1 Let $0<p_{1} \leq u_{1}<\infty$ and $0<p_{2} \leq u_{2}<\infty$. Then the embedding

$$
m_{u_{1}, p_{1}} \hookrightarrow m_{u_{2}, p_{2}}
$$

is continuous if and only if the following conditions hold:

$$
u_{1} \leq u_{2} \quad \text { and } \quad \frac{p_{2}}{u_{2}} \leq \frac{p_{1}}{u_{1}} .
$$

The embedding (3.1) is never compact. 
Proof Step 1. First we prove the sufficiency of the conditions. If $u_{1}=u_{2}$, then $p_{2} \leq p_{1}$, and by the Hölder inequality we get for any $j \in \mathbb{N}_{0}$ and $m \in \mathbb{Z}^{d}$,

$$
\left|Q_{-j, m}\right|^{-\frac{1}{p_{2}}}\left(\sum_{k: Q_{0, k} \subset Q_{-j, m}}\left|\lambda_{k}\right|^{p_{2}}\right)^{\frac{1}{p_{2}}} \leq\left|Q_{-j, m}\right|^{-\frac{1}{p_{1}}}\left(\sum_{k: Q_{0, k} \subset Q_{-j, m}}\left|\lambda_{k}\right|^{p_{1}}\right)^{\frac{1}{p_{1}}} .
$$

So (3.1) holds for $u_{1}=u_{2}$.

Let now $u_{1}<u_{2}$. If $\frac{p_{1}}{u_{1}}=\frac{p_{2}}{u_{2}}$, then for any $j \in \mathbb{N}_{0}$ and $m \in \mathbb{Z}^{d}$, we get

$$
\begin{aligned}
2^{j d\left(\frac{1}{u_{2}}-\frac{1}{p_{2}}\right)} & \left(\sum_{k: Q_{0, k} \subset Q_{-j, m}}\left|\lambda_{k}\right|^{p_{2}}\right)^{\frac{1}{p_{2}}} \\
& \leq \sup _{k: Q_{0, k} \subset Q_{-j, m}}\left|\lambda_{k}\right|^{1-\frac{p_{1}}{p_{2}}}\left[2^{j d\left(\frac{1}{u_{1}}-\frac{1}{p_{1}}\right)}\left(\sum_{k: Q_{0, k} \subset Q_{-j, m}}\left|\lambda_{k}\right|^{p_{1}}\right)^{\frac{1}{p_{1}}}\right]^{\frac{p_{1}}{p_{2}}} .
\end{aligned}
$$

The last inequality implies that

$$
\left\|\lambda\left|m_{u_{2}, p_{2}}\|\leq\| \lambda\right| m_{u_{1}, p_{1}}\right\|^{\frac{p_{1}}{p_{2}}}\left\|\lambda\left|\ell_{\infty}\left\|^{1-\frac{p_{1}}{p_{2}}} \leq\right\| \lambda\right| m_{u_{1}, p_{1}}\right\| .
$$

If $\frac{p_{2}}{u_{2}}<\frac{p_{1}}{u_{1}}$, then $p_{1}>\widetilde{p_{1}}=u_{1} \frac{p_{2}}{u_{2}}$. So the statement follows from (3.3) and the monotonicity, see Proposition 2.3(ii).

Step 2. We consider the necessity of the conditions.

Substep 2.1. First we assume that $u_{2}<u_{1}$. For any $j \in \mathbb{N}$, we put $m_{j}=$ $\left(2^{2 j}, 0, \ldots, 0\right) \in \mathbb{Z}^{d}$. We put

$$
\lambda_{k}= \begin{cases}\left|Q_{-j, m_{j}}\right|^{-\frac{1}{u_{1}}} & \text { if } Q_{0, k} \subset Q_{-j, m_{j}} \\ 0 & \text { otherwise. }\end{cases}
$$

Then straightforward calculation shows that $\left\|\lambda \mid m_{u_{1}, p_{1}}\right\|=1$. On the other hand,

$$
\sup _{j \in \mathbb{N}_{0}}\left|Q_{-j, m_{j}}\right|^{\frac{1}{u_{2}}-\frac{1}{p_{2}}}\left(\sum_{k: Q_{0, k} \subset Q_{-j, m_{j}}}\left|\lambda_{k}\right|^{p_{2}}\right)^{1 / p_{2}}=\sup _{j \in \mathbb{N}_{0}}\left|Q_{-j, m_{j}}\right|^{\frac{1}{u_{2}}-\frac{1}{u_{1}}}=\infty .
$$

So $\left\{\lambda_{k}\right\}_{k}$ does not belong to $m_{u_{2}, p_{2}}$.

Substep 2.2. Now we assume that $u_{1} \leq u_{2}$ and $\frac{p_{1}}{u_{1}}<\frac{p_{2}}{u_{2}}$, in particular, $\frac{p_{1}}{u_{1}}<1$. For any $j \in \mathbb{N}$, we put

$$
k_{j}=\left\lfloor 2^{d j\left(1-\frac{p_{1}}{u_{1}}\right)}\right\rfloor
$$


recall $\lfloor x\rfloor=\max \{l \in \mathbb{Z}: l \leq x\}$. Then $1 \leq k_{v}<2^{d v}$ and

$$
k_{j} \leq c_{p_{1} q_{1}} 2^{d(j-v)} k_{v}, \quad \text { if } \quad 1 \leq v<j .
$$

For convenience let us assume that $c_{p_{1} q_{1}}=1$ (otherwise the argument below has to be modified in an obvious way). For any $j \in \mathbb{N}$, we define a sequence $\lambda^{(j)}=\left\{\lambda_{k}^{(j)}\right\}_{k}$ in the following way. We assume that $k_{j}$ elements of the sequence equal 1 and the rest is equal to 0 . If $Q_{0, k} \nsubseteq Q_{-j, 0}$, then we put $\lambda_{k}^{(j)}=0$. Moreover, because of the inequality (3.4), we can choose the elements that equal 1 in such a way that the following property holds:

$$
\text { if } Q_{-v, \ell} \subseteq Q_{-j, 0} \text { and } Q_{-v, \ell}=\bigcup_{i=1}^{2^{d v}} Q_{0, m_{i}},
$$

then at most $k_{v}$ elements $\lambda_{0, k_{i}}^{(j)}$ equal 1 .

By construction, if $Q_{-v, \ell} \subseteq Q_{-j, 0}$, then

$$
\sum_{k: Q_{0, k} \subset Q_{-v, \ell}}\left|\lambda_{0, k}^{(j)}\right|^{q_{1}} \leq k_{v} \leq 2^{d v\left(1-\frac{p_{1}}{u_{1}}\right)}
$$

and the last sum is equal to $k_{v}$ if $v=j$. Thus

$$
\left\|\lambda^{(j)} \mid m_{u_{1}, p_{1}}\right\| \leq 1
$$

Furthermore, the assumption $0<\frac{p_{1}}{u_{1}}<\frac{p_{2}}{u_{2}} \leq 1$ implies that

$$
\frac{\left\lfloor 2^{d j\left(1-\frac{p_{1}}{u_{1}}\right)}\right\rfloor}{2^{d j\left(1-\frac{p_{2}}{u_{2}}\right)}} \longrightarrow \infty \text { if } \quad j \rightarrow \infty .
$$

So for any $N \in \mathbb{N}$, there exists a number $j_{N} \in \mathbb{N}$ such that

$$
N 2^{d j_{N}\left(1-\frac{p_{2}}{u_{2}}\right)} \leq\left\lfloor 2^{d j_{N}\left(1-\frac{p_{1}}{q_{1}}\right)}\right\rfloor=k_{j_{N}}=\sum_{k: Q_{0, k} \subset Q_{-j_{N}, 0}}\left|\lambda_{0, k}^{\left(j_{N}\right)}\right|^{p_{2}}
$$

But this immediately implies that

$$
N^{1 / p_{2}} \leq\left\|\lambda^{\left(j_{N}\right)} \mid m_{u_{2}, p_{2}}\right\|
$$

However, since we assume that the embedding (3.1) holds, there is a positive constant $c>0$ such that

$$
\left\|\lambda^{(j)}\left|m_{u_{2}, p_{2}}\|\leq c\| \lambda^{(j)}\right| m_{u_{1}, p_{1}}\right\| \leq c \quad \text { for any } \quad \lambda^{(j)} \in m_{u_{1}, p_{1}} .
$$


In view of the last inequalities (3.6) and (3.7), we get

$$
N^{1 / p_{2}} \leq\left\|\lambda^{\left(j_{N}\right)}\left|m_{u_{2}, p_{2}}\|\leq C\| \lambda^{\left(j_{N}\right)}\right| m_{u_{1}, p_{1}}\right\| \leq C,
$$

and this leads to a contradiction for large $N$.

Step 3. The noncompactness of (3.1) immediately follows from Proposition 2.3,

$$
\ell_{u_{1}} \hookrightarrow m_{u_{1}, p_{1}} \hookrightarrow m_{u_{2}, p_{2}} \hookrightarrow \ell_{\infty}
$$

and the noncompactness of $\ell_{u_{1}} \hookrightarrow \ell_{\infty}$.

Corollary 3.2 Let $0<p_{1} \leq u_{1}<\infty$ and $0<p_{2} \leq u_{2}<\infty$. Then $m_{u_{1}, p_{1}}=m_{u_{2}, p_{2}}$ (in the sense of equivalent norms) if and only if $u_{1}=u_{2}$ and $p_{1}=p_{2}$.

Proof This follows immediately from Theorem 3.1.

Corollary 3.3 Let $0<p_{1} \leq u_{1}<\infty$ and $0<p_{2} \leq u_{2}<\infty$.

(i) Then $m_{u_{1}, p_{1}} \hookrightarrow \ell_{u_{2}}$ if and only if $u_{1} \leq u_{2}$ and $p_{1}=u_{1}$, that is, if and only if $m_{u_{1}, p_{1}}=\ell_{u_{1}}$ and $\ell_{u_{1}} \hookrightarrow \ell_{u_{2}}$.

(ii) Then $\ell_{u_{1}} \hookrightarrow m_{u_{2}, p_{2}}$ if and only if $u_{1} \leq u_{2}$, that is, if and only if $\ell_{u_{1}} \hookrightarrow \ell_{u_{2}}$.

Proof This follows immediately from Theorem 3.1.

Remark 3.4 Let us mention the following essential feature: if $0<p<u<\infty$, that is, we are in the proper Morrey situation, then there is never an embedding into any space $\ell_{r}$ whenever $0<r<\infty$, but we always have $m_{u, p} \hookrightarrow \ell_{\infty}$, in view of Proposition 2.3(iii) and Corollary 3.3(i).

Remark 3.5 We briefly want to compare Theorem 3.1 with forerunners in [16] and in the parallel setting of Morrey function spaces.

In [16, Prop. 2.4] the one-dimensional counterpart of Theorem 3.1 can be found in the case when (in our notation) $1 \leq p_{2} \leq p_{1} \leq u_{1}=u_{2}<\infty$, with some discussion about the sharpness of that result. Obviously condition (3.2) is automatically satisfied in this case. The method of their proof in [16] is different from ours.

We turn to function spaces and first consider spaces $\mathcal{M}_{u, p}(Q)$ defined on a cube $Q$, where (1.1) has to be adapted appropriately. Then by a result of Piccinini in [34], see also [35], for $0<p_{i} \leq u_{i}<\infty, i=1,2$,

$$
\mathcal{M}_{u_{1}, p_{1}}(Q) \hookrightarrow \mathcal{M}_{u_{2}, p_{2}}(Q) \quad \text { if and only if } \quad p_{2} \leq p_{1} \quad \text { and } \quad u_{2} \leq u_{1} \text {. }
$$

This result was extended to $\mathbb{R}^{d}$ by Rosenthal in [38], reading as

$$
\mathcal{M}_{u_{1}, p_{1}}\left(\mathbb{R}^{d}\right) \hookrightarrow \mathcal{M}_{u_{2}, p_{2}}\left(\mathbb{R}^{d}\right) \quad \text { if and only if } \quad p_{2} \leq p_{1} \leq u_{1}=u_{2}
$$

So a similar diversity as in the classical $L_{p}$-setting (spaces on bounded domains versus $\mathbb{R}^{d}$ versus sequence spaces $\ell_{p}$ ) is obvious.

What is, however, more surprising is the similarity with our result [18, Thm. 3.2] in the context of sequence spaces $n_{u, p, q}^{s}$ appropriate for smoothness Morrey spaces. 
In the limiting situation $s_{1}-\frac{d}{u_{1}}=s_{2}-\frac{d}{u_{2}}$ (and in adapted notation), we have shown that

$$
n_{u_{1}, p_{1}, q_{1}}^{s_{1}} \hookrightarrow n_{u_{2}, p_{2}, q_{2}}^{s_{2}}
$$

if and only if (3.2) and $q_{1} \leq q_{2}$.

\section{A Pre-dual of $m_{u, p}$}

Results concerning (pre-)dual spaces in the setting of Morrey function spaces have some history; we refer to $[5,20,58]$ and, more recently, to [2] in this respect. We rely on the paper [40, Sect. 4], where further discussion can also be found.

Definition 4.1 Let $1 \leq p<u<\infty$ and $\frac{1}{p}+\frac{1}{p^{\prime}}=1$, as usual. For any $j \in \mathbb{N}_{0}$, we define $\mathcal{X}_{u, p}^{(j)}=\mathcal{X}_{u, p}^{(j)}\left(\mathbb{Z}^{d}\right)$ by

$$
\begin{aligned}
& \mathcal{X}_{u, p}^{(j)}\left(\mathbb{Z}^{d}\right)=\left\{\lambda=\left\{\lambda_{k}\right\}_{k \in \mathbb{Z}^{d}} \subset \mathbb{C}:\right. \\
&\left.\|\lambda\|_{u, p}^{(j)}=2^{j d\left(\frac{1}{p}-\frac{1}{u}\right)} \sum_{m \in \mathbb{Z}^{d}}\left(\sum_{k: Q_{0, k} \subset Q_{-j, m}}\left|\lambda_{k}\right|^{p^{\prime}}\right)^{\frac{1}{p^{\prime}}}<\infty\right\} .
\end{aligned}
$$

Lemma 4.2 Let $1 \leq p<u<\infty$ and $j \in \mathbb{N}_{0}$. Then

$$
\mathcal{X}_{u, p}^{(j)}\left(\mathbb{Z}^{d}\right)=\ell_{1}\left(\mathbb{Z}^{d}\right)
$$

(in the sense of equivalent norms). Moreover, the embedding

$$
\operatorname{id}_{j}: \mathcal{X}_{u, p}^{(j)}\left(\mathbb{Z}^{d}\right) \hookrightarrow \ell_{u^{\prime}}\left(\mathbb{Z}^{d}\right)
$$

satisfies

$$
\left\|\operatorname{id}_{j}: \mathcal{X}_{u, p}^{(j)}\left(\mathbb{Z}^{d}\right) \hookrightarrow \ell_{u^{\prime}}\left(\mathbb{Z}^{d}\right)\right\|=1 .
$$

Proof We begin with (4.2). Note that

$$
\left(\sum_{k: Q_{0, k} \subset Q_{-j, m}}\left|\lambda_{k}\right|^{p^{\prime}}\right)^{\frac{1}{p^{\prime}}} \leq \sum_{k: Q_{0, k} \subset Q_{-j, m}}\left|\lambda_{k}\right| \leq 2^{j \frac{d}{p}}\left(\sum_{k: Q_{0, k} \subset Q_{-j, m}}\left|\lambda_{k}\right|^{p^{\prime}}\right)^{\frac{1}{p^{\prime}}} .
$$


Hence, by definition,

$$
\begin{aligned}
\|\lambda\|_{u, p}^{(j)} & =2^{j d\left(\frac{1}{p}-\frac{1}{u}\right)} \sum_{m \in \mathbb{Z}^{d}}\left(\sum_{k: Q_{0, k} \subset Q_{-j, m}}\left|\lambda_{k}\right|^{p^{\prime}}\right)^{\frac{1}{p^{\prime}}} \\
& \geq 2^{j d\left(\frac{1}{p}-\frac{1}{u}\right)} \sum_{m \in \mathbb{Z}^{d}}\left(\sum_{k: Q_{0, k} \subset Q_{-j, m}}\left|\lambda_{k}\right|\right) 2^{-j \frac{d}{p}} \\
& =2^{-j \frac{d}{u}} \sum_{k \in \mathbb{Z}^{d}}\left|\lambda_{k}\right|=2^{-j d \frac{1}{u}}\left\|\lambda \mid \ell_{1}\right\|,
\end{aligned}
$$

hence $\mathcal{X}_{u, p}^{(j)} \hookrightarrow \ell_{1}$. Conversely,

$$
\begin{aligned}
\|\lambda\|_{u, p}^{(j)} & =2^{j d\left(\frac{1}{p}-\frac{1}{u}\right)} \sum_{m \in \mathbb{Z}^{d}}\left(\sum_{k: Q_{0, k} \subset Q_{-j, m}}\left|\lambda_{k}\right|^{p^{\prime}}\right)^{\frac{1}{p^{\prime}}} \\
& \leq 2^{j d\left(\frac{1}{p}-\frac{1}{u}\right)} \sum_{k \in \mathbb{Z}^{d}}\left|\lambda_{k}\right|=2^{j d\left(\frac{1}{p}-\frac{1}{u}\right)}\left\|\lambda \mid \ell_{1}\right\|,
\end{aligned}
$$

which results in $\ell_{1} \hookrightarrow \mathcal{X}_{u, p}^{(j)}$ and thus finishes the proof of (4.2).

Similarly to (4.4), we obtain for the embedding $\mathrm{id}_{j}$,

$$
\begin{aligned}
\|\lambda\|_{u, p}^{(j)} & =2^{j d\left(\frac{1}{p}-\frac{1}{u}\right)} \sum_{m \in \mathbb{Z}^{d}}\left(\sum_{k: Q_{0, k} \subset Q_{-j, m}}\left|\lambda_{k}\right|^{p^{\prime}}\right)^{\frac{1}{p^{\prime}}} \\
& \geq 2^{j d\left(\frac{1}{p}-\frac{1}{u}\right)} \sum_{m \in \mathbb{Z}^{d}}\left(\sum_{k: Q_{0, k} \subset Q_{-j, m}}\left|\lambda_{k}\right|^{u^{\prime}}\right)^{\frac{1}{u^{\prime}}} 2^{-j d\left(\frac{1}{u^{\prime}}-\frac{1}{p^{\prime}}\right)} \\
& \geq 2^{j d\left(\frac{1}{p}-\frac{1}{u}-\frac{1}{p}+\frac{1}{u}\right)}\left(\sum_{k \in \mathbb{Z}^{d}}\left|\lambda_{k}\right|^{u^{\prime}}\right)^{\frac{1}{u^{\prime}}}=\left\|\lambda \mid \ell_{u^{\prime}}\right\|
\end{aligned}
$$

since $p^{\prime}>u^{\prime}>1$. Thus $\left\|\mathrm{id}_{j}\right\| \leq 1$. Now let $m_{0} \in \mathbb{Z}^{d}$ be fixed, and consider $\lambda^{0}=\left\{\lambda_{k}^{0}\right\}_{k \in \mathbb{Z}^{d}}$ given by

$$
\lambda_{k}^{0}= \begin{cases}2^{-j \frac{d}{u^{\prime}}} & \text { if } Q_{0, k} \subset Q_{-j, m_{0}} \\ 0 & \text { otherwise }\end{cases}
$$


Thus $\left\|\left.\lambda^{0}\left|\ell_{u^{\prime}} \|=2^{-j \frac{d}{u^{\prime}}}\right| Q_{-j, m_{0}}\right|^{\frac{1}{u^{\prime}}}=1\right.$, and

$$
\begin{aligned}
\left\|\lambda^{0}\right\|_{u, p}^{(j)} & =2^{j d\left(\frac{1}{p}-\frac{1}{u}\right)} \sum_{m \in \mathbb{Z}^{d}}\left(\sum_{k: Q_{0, k} \subset Q_{-j, m}}\left|\lambda_{k}\right|^{p^{\prime}}\right)^{\frac{1}{p^{\prime}}}=2^{j d\left(\frac{1}{p}-\frac{1}{u}\right)} 2^{-j \frac{d}{u^{\prime}}\left|Q_{-j, m_{0}}\right|^{\frac{1}{p^{\prime}}}} \\
& =2^{j d\left(\frac{1}{p}-\frac{1}{u}-\frac{1}{u^{\prime}}+\frac{1}{p^{\prime}}\right)}=1,
\end{aligned}
$$

such that finally $\left\|\mathrm{id}_{j}\right\| \geq 1$. This completes the proof of (4.3).

Now we combine the above sequence spaces $\mathcal{X}_{u, p}^{(j)}\left(\mathbb{Z}^{d}\right)$ at level $j \in \mathbb{N}_{0}$ as follows.

Definition 4.3 Let $1 \leq p<u<\infty$. We define $\mathcal{X}_{u, p}=\mathcal{X}_{u, p}\left(\mathbb{Z}^{d}\right)$ by

$$
\begin{aligned}
\mathcal{X}_{u, p}\left(\mathbb{Z}^{d}\right)=\left\{\lambda=\left\{\lambda_{k}\right\}_{k \in \mathbb{Z}^{d}} \subset \mathbb{C}: \text { for any } j \in \mathbb{N}_{0} \text { there exists } \lambda^{(j)} \in \mathcal{X}_{u, p}^{(j)}\left(\mathbb{Z}^{d}\right)\right. \\
\text { such that } \left.\lambda=\sum_{j=0}^{\infty} \lambda^{(j)}, \text { and } \sum_{j=0}^{\infty}\left\|\lambda^{(j)}\right\|_{u, p}^{(j)}<\infty\right\}
\end{aligned}
$$

equipped with the norm

$$
\left\|\lambda \mid \mathcal{X}_{u, p}\right\|=\inf \sum_{j=0}^{\infty}\left\|\lambda^{(j)}\right\|_{u, p}^{(j)},
$$

where the infimum is taken over all admitted decompositions of $\lambda$ according to (4.6).

Proposition 4.4 Let $1 \leq p<u<\infty$.

(i) Then

$$
\ell_{1}\left(\mathbb{Z}^{d}\right) \hookrightarrow \mathcal{X}_{u, p}\left(\mathbb{Z}^{d}\right) \hookrightarrow \ell_{u^{\prime}}\left(\mathbb{Z}^{d}\right)
$$

and

$$
\| \text { id }: \ell_{1}\left(\mathbb{Z}^{d}\right) \hookrightarrow \mathcal{X}_{u, p}\left(\mathbb{Z}^{d}\right)\|=\| \text { id }: \mathcal{X}_{u, p}\left(\mathbb{Z}^{d}\right) \hookrightarrow \ell_{u^{\prime}}\left(\mathbb{Z}^{d}\right) \|=1
$$

(ii) Let for $n \in \mathbb{Z}^{d}$, $e^{(n)}=\left\{e_{k}^{(n)}\right\}_{k \in \mathbb{Z}^{d}}$ be given by

$$
e_{k}^{(n)}= \begin{cases}1 & \text { if } n=k \\ 0 & \text { otherwise }\end{cases}
$$

The system $\left\{e^{(n)}\right\}_{n \in \mathbb{Z}^{d}}$ forms a normalized unconditional basis in $\mathcal{X}_{u, p}\left(\mathbb{Z}^{d}\right)$. (iii) $\mathcal{X}_{u, p}\left(\mathbb{Z}^{d}\right)$ is a separable Banach space. 
Proof Step 1. Let $\lambda \in \ell_{1}$. Then Lemma 4.2 applied with $j=0$, in particular (4.2), imply that we obtain an admitted representation of $\lambda$ in (4.6) choosing $\lambda^{(0)}=\lambda \in \mathcal{X}_{u, p}^{(0)}$ and $\lambda^{(j)}=0, j \in \mathbb{N}$. This ensures $\lambda \in \mathcal{X}_{u, p}$ and, in view of (4.7) and (4.5),

$$
\left\|\lambda\left|\mathcal{X}_{u, p}\|\leq\| \lambda\left\|_{u, p}^{(0)} \leq\right\| \lambda\right| \ell_{1}\right\|
$$

Thus $\|$ id $: \ell_{1}\left(\mathbb{Z}^{d}\right) \hookrightarrow \mathcal{X}_{u, p}\left(\mathbb{Z}^{d}\right) \| \leq 1$. If $\lambda \in \mathcal{X}_{u, p}$, then there exists a decomposition according to (4.6), and we can conclude

$$
\left\|\lambda\left|\ell_{u^{\prime}}\left\|\leq \sum_{j=0}^{\infty}\right\| \lambda^{(j)}\right| \ell_{u^{\prime}}\right\| \leq \sum_{j=0}^{\infty}\left\|\lambda^{(j)}\right\|_{u, p}^{(j)},
$$

where we applied (4.3). Taking the infimum over all possible representations according to (4.6), we get by (4.7) that

$$
\left\|\lambda\left|\ell_{u^{\prime}}\|\leq\| \lambda\right| \mathcal{X}_{u, p}\right\|, \quad \text { and thus } \quad \| \text { id }: \mathcal{X}_{u, p}\left(\mathbb{Z}^{d}\right) \hookrightarrow \ell_{u^{\prime}}\left(\mathbb{Z}^{d}\right) \| \leq 1
$$

To complete the proof of (i), we have to show the converse inequalities in (4.8). However, $\mathcal{X}_{u, p}^{(j)} \hookrightarrow \mathcal{X}_{u, p}$ for any $j \in \mathbb{N}_{0}$ with $\|$ id $: \mathcal{X}_{u, p}^{(j)} \hookrightarrow \mathcal{X}_{u, p} \| \leq 1$, such that (4.3) yields

$$
1=\left\|\operatorname{id}: \mathcal{X}_{u, p}^{(j)} \hookrightarrow \ell_{u^{\prime}}\right\| \leq\left\|\operatorname{id}: \mathcal{X}_{u, p} \hookrightarrow \ell_{u^{\prime}}\right\|
$$

confirming the latter equality in (4.8). Now we are done, since

$1=\left\|\mathrm{id}: \ell_{1} \hookrightarrow \ell_{u^{\prime}}\right\| \leq\left\|\mathrm{id}: \ell_{1} \hookrightarrow \mathcal{X}_{u, p}\right\|\left\|\mathrm{id}: \mathcal{X}_{u, p} \hookrightarrow \ell_{u^{\prime}}\right\|=\left\|\mathrm{id}: \ell_{1} \hookrightarrow \mathcal{X}_{u, p}\right\|$.

Step 2. Concerning (ii), one can easily calculate that $\left\|e^{(n)} \mid \mathcal{X}_{u, p}\right\|=1$ and that the system is complete in $\mathcal{X}_{u, p}$. So the statement follows from the trivial inequality

$$
\left\|\sum_{|n| \leq \ell} \varepsilon_{n} \lambda_{n} e^{(n)}\left|\mathcal{X}_{u, p}\|\leq\| \sum_{|n| \leq \ell} \lambda_{n} e^{(n)}\right| \mathcal{X}_{u, p}\right\|,
$$

$\varepsilon_{n}= \pm 1, \lambda_{n} \in \mathbb{C}$, cf., e.g., [19, Theorem 6.7].

Step 3. The proof of (iii) is standard.

Proposition 4.5 Let $1 \leq p<u<\infty$. Then $\mathcal{X}_{u, p}\left(\mathbb{Z}^{d}\right)$ is a pre-dual space of $m_{u, p}\left(\mathbb{Z}^{d}\right)$. 
Proof Let $\mu \in m_{u, p}, \lambda \in \mathcal{X}_{u, p}\left(\mathbb{Z}^{d}\right)$ and let $\lambda=\sum_{j=0}^{\infty} \lambda^{(j)}, \lambda^{(j)} \in \mathcal{X}_{u, p}^{(j)}$. Then

$$
\begin{aligned}
\left|\sum_{k \in \mathbb{Z}^{d}} \lambda_{k} \mu_{k}\right| & \leq \sum_{j=0}^{\infty} \sum_{k \in \mathbb{Z}^{d}}\left|\lambda_{k}^{(j)} \mu_{k}\right| \\
& =\sum_{j=0}^{\infty} \sum_{m \in \mathbb{Z}^{d}} \sum_{k: Q_{0, k} \subset Q_{-j, m}}\left|\lambda_{k}^{(j)} \mu_{k}\right| \\
& \leq \sum_{j=0}^{\infty} \sum_{m \in \mathbb{Z}^{d}}\left(\sum_{k: Q_{0, k} \subset Q_{-j, m}}\left|\mu_{k}\right|^{p}\right)^{\frac{1}{p}}\left(\sum_{k: Q_{0, k} \subset Q_{-j, m}}\left|\lambda_{k}^{(j)}\right|^{p^{\prime}}\right)^{\frac{1}{p^{\prime}}} \\
& \leq\left\|\mu \mid m_{u, p}\right\| \sum_{j=0}^{\infty} 2^{d j\left(\frac{1}{p}-\frac{1}{u}\right)} \sum_{m \in \mathbb{Z}^{d}}\left(\sum_{k: Q_{0, k} \subset Q_{-j, m}}\left|\lambda_{k}^{(j)}\right|^{p^{\prime}}\right)^{\frac{1}{p^{\prime}}} \\
& \leq\left\|\mu \mid m_{u, p}\right\| \sum_{j=0}^{\infty}\left\|\lambda^{(j)}\right\|_{u, p}^{(j)} .
\end{aligned}
$$

Taking the infimum over all representations of $\lambda$, we get

$$
\left|\sum_{k \in \mathbb{Z}^{d}} \lambda_{k} \mu_{k}\right| \leq\left\|\mu\left|m_{u, p}\|\| \lambda\right| \mathcal{X}_{u, p}\right\| .
$$

On the other hand, if $f \in\left(\mathcal{X}_{u, p}\left(\mathbb{Z}^{d}\right)\right)^{\prime}$, then

$$
|f(\lambda)| \leq\|f\|\left\|\lambda \mid \mathcal{X}_{u, p}\right\|
$$

where $\|f\|=\sup _{\left\|\lambda \mid \mathcal{X}_{u, p}\right\|=1}|f(\lambda)|$, as usual. For any dyadic cube $Q_{-v, m}, v \in \mathbb{N}_{0}$, we take

$$
\lambda^{(\nu, m)}=\sum_{k: Q_{0, k} \subset Q_{-v, m}} 2^{\nu d\left(\frac{1}{p}-\frac{1}{u}\right)} \lambda_{k} e^{(k)} .
$$

Then $\lambda^{(v, m)} \in \mathcal{X}_{u, p}$ if and only if $\left\{\lambda_{k}\right\}_{k \in \mathbb{Z}^{d}} \in \ell_{p^{\prime}}^{2^{v d}}\left(Q_{-v, m}\right)$ and $\left\|\lambda^{(v, m)} \mid \mathcal{X}_{u, p}\right\| \leq$ $\left\|\left\{\lambda_{k}\right\}_{k} \mid \ell_{p^{\prime}}^{2^{v d}}\left(Q_{-v, m}\right)\right\|$. Moreover,

$$
\begin{aligned}
\left|\sum_{k: Q_{0, k} \subset Q_{-v, m}} \lambda_{k} 2^{\nu d\left(\frac{1}{p}-\frac{1}{u}\right)} f\left(e^{(k)}\right)\right| & =\left|f\left(\lambda^{(v, m)}\right)\right| \leq\|f\|\left\|\lambda^{(v, m)} \mid \mathcal{X}_{u, p}\right\| \\
& \leq\|f\|\left\|\left\{\lambda_{k}\right\}_{k} \mid \ell_{p^{\prime}}^{2^{d v}}\left(Q_{-v, m}\right)\right\| .
\end{aligned}
$$


By duality for the $\ell_{p}$ spaces, we get $\left\{2^{\nu d\left(\frac{1}{p}-\frac{1}{u}\right)} f\left(e^{(k)}\right)\right\}_{k} \in \ell_{p}^{2^{d v}}\left(Q_{-v, m}\right)$ and

$$
\begin{aligned}
\left|Q_{-v, m}\right|^{\frac{1}{u}-\frac{1}{p}}\left(\sum_{k: Q_{0, k} \subset Q_{-v, m}}\left|f\left(e^{(k)}\right)\right|^{p}\right)^{\frac{1}{p}} & =\left\|\left\{2^{v d\left(\frac{1}{p}-\frac{1}{u}\right)} f\left(e^{(k)}\right)\right\}_{k} \mid \ell_{p}^{2^{d v}}\left(Q_{-v, m}\right)\right\| \\
& \leq\|f\| .
\end{aligned}
$$

So $\left\{f\left(e^{(k)}\right)\right\}_{k} \in m_{u, p}\left(\mathbb{Z}^{d}\right)$ and $\left\|\left\{f\left(e^{(k)}\right)\right\}_{k} \mid m_{u, p}\right\| \leq\|f\|$.

Next we define a closed proper subspace of $m_{u, p}$ as follows. Let $c_{00}$ denote the finite sequences in $\mathbb{C}$, that is, sequences that possess only finitely many nonvanishing elements. We define $m_{u, p}^{00}=m_{u, p}^{00}\left(\mathbb{Z}^{d}\right)$ to be the closure of $c_{00}$ in $m_{u, p}$,

$$
m_{u, p}^{00}=\overline{c_{00}}\left\|\cdot \mid m_{u, p}\right\| .
$$

Obviously $m_{u, p}^{00}$ is separable. We shall prove below that $\mathcal{X}_{u, p}$ is the dual space of $m_{u, p}^{00}$. We begin with some general properties. For that reason, let us denote by $m_{u, p}^{0}=$ $m_{u, p}^{0}\left(\mathbb{Z}^{d}\right)$ the subspace of null sequences which belong to $m_{u, p}$,

$$
m_{u, p}^{0}=m_{u, p} \cap c_{0}
$$

Then we have the following basic properties.

Lemma 4.6 Let $0<p<u<\infty$. Then $m_{u, p}^{0}$ and $m_{u, p}^{00}$ are proper closed subspaces of $m_{u, p}$, with

$$
m_{u, p}^{00} \subsetneq m_{u, p}^{0} \subsetneq m_{u, p} .
$$

Proof By definition, $m_{u, p}^{00}$ is a closed subspace of $m_{u, p}$. The fact that $m_{u, p}^{0} \subsetneq m_{u, p}$ is a proper subspace of $m_{u, p}$ follows from Proposition 2.3(v). We show that $m_{u, p}^{0}$ is closed in $m_{u, p}$,

$$
m_{u, p}^{0}={\overline{m_{u, p}^{0}}}^{\left\|\cdot \mid m_{u, p}\right\|}
$$

Clearly $m_{u, p}^{0} \subseteq \overline{m_{u, p}^{0}}\left\|\cdot \mid m_{u, p}\right\|$, so we have to verify the converse inclusion. Let $\lambda=$ $\left\{\lambda_{k}\right\}_{k \in \mathbb{Z}^{d}} \in \overline{m_{u, p}^{0}}\left\|\cdot \mid m_{u, p}\right\|$ and $\varepsilon>0$ be arbitrary. Then, by definition, there exists some $\mu=\left\{\mu_{k}\right\}_{k \in \mathbb{Z}^{d}} \in m_{u, p}^{0}$ such that

$$
\left\|\mu-\left.\lambda\left|m_{u, p} \|=\sup _{j \in \mathbb{N}_{0}, m \in \mathbb{Z}^{d}}\right| Q_{-j, m}\right|^{\frac{1}{u}-\frac{1}{p}}\left(\sum_{k: Q_{0, k} \subset Q_{-j, m}}\left|\mu_{k}-\lambda_{k}\right|^{p}\right)^{\frac{1}{p}}<\varepsilon .\right.
$$


Now $\mu \in m_{u, p}^{0} \subset m_{u, p}$ and $m_{u, p}$ is complete, thus $\lambda \in m_{u, p}$. Moreover, applying (4.9) with $j=0$ implies that

$$
\left\|\mu-\lambda\left|\ell_{\infty} \|=\sup _{k \in \mathbb{Z}^{d}}\right| \mu_{k}-\lambda_{k} \mid<\varepsilon\right.
$$

However, $\mu \in m_{u, p}^{0} \subset c_{0}$ thus leads to $\lambda \in c_{0}$. So finally $\lambda \in m_{u, p} \cap c_{0}=m_{u, p}^{0}$.

It remains to verify that $m_{u, p}^{00} \subsetneq m_{u, p}^{0}$. First note that, by definition, $m_{u, p}^{00} \subseteq m_{u, p}^{0}$. Now consider special lattice points $m_{j}=\left(2^{2 j}, 0, \ldots, 0\right) \in \mathbb{Z}^{d}, j \in \mathbb{N}_{0}$, and put

$$
\lambda_{k}= \begin{cases}2^{-j \frac{d}{u}} & \text { if } Q_{0, k} \subset Q_{-j, m_{j}}, \\ 0 & \text { otherwise }\end{cases}
$$

Then $\lambda \in c_{0} \cap m_{u, p}=m_{u, p}^{0}$, but obviously $\lambda \notin m_{u, p}^{00}$.

Remark 4.7 The above result sheds some further light on the difference of the two norms $\left\|\cdot \mid \ell_{\infty}\right\|$ and $\left\|\cdot \mid m_{u, p}\right\|$, since in the classical setting,

$$
\overline{c_{00}}\left\|\cdot \ell_{\infty}\right\|=c_{0}
$$

is well known, in contrast to $m_{u, p}^{00} \subsetneq m_{u, p}^{0}$.

We need the following lemma.

Lemma 4.8 Let $0<p<u<\infty$ and $\lambda \in m_{u, p}^{00}\left(\mathbb{Z}^{d}\right)$. Then there exists a dyadic cube $Q(\lambda)$ such that

$$
\left\|\left.\lambda\left|m_{u, p} \|=\right| Q(\lambda)\right|^{\frac{1}{u}-\frac{1}{p}}\left(\sum_{k: Q_{0, k} \subset Q(\lambda)}\left|\lambda_{k}\right|^{p}\right)^{\frac{1}{p}} .\right.
$$

Proof For any sequence $\lambda \in m_{u, p}$ and any dyadic cube $Q$, we shall denote by $\left.\lambda\right|_{Q}$ the restriction of $\lambda$ to $Q$, i.e., $\left(\left.\lambda\right|_{Q}\right)_{k}=\lambda_{k}$ if $Q_{0, k} \subset Q$ and $\left(\left.\lambda\right|_{Q}\right)_{k}=0$ otherwise. Without loss of generality, we may assume $\lambda \not \equiv 0$.

We choose a positive number $\varepsilon<1-2^{d\left(\frac{1}{u}-\frac{1}{p}\right)}$. If $\lambda \in m_{u, p}^{00}$, then there exists a dyadic cube $\widetilde{Q}$ such that

$$
\left\|\lambda-\left.\lambda\right|_{\widetilde{Q}}\left|m_{u, p}\|\leq \varepsilon\| \lambda\right| m_{u, p}\right\|
$$

If $Q \cap \widetilde{Q}=\emptyset$, then

$$
\begin{aligned}
|Q|^{\frac{1}{u}-\frac{1}{p}}\left(\sum_{k: Q_{0, k} \subset Q}\left|\lambda_{k}\right|^{p}\right)^{\frac{1}{p}} & \leq|Q|^{\frac{1}{u}-\frac{1}{p}}\left(\sum_{k: Q_{0, k} \subset Q}\left|\lambda_{k}-(\lambda \mid \widetilde{Q})_{k}\right|^{p}\right)^{\frac{1}{p}} \\
& \leq \varepsilon\left\|\lambda\left|m_{u, p}\|<\| \lambda\right| m_{u, p}\right\| .
\end{aligned}
$$


If $\widetilde{Q} \varsubsetneqq Q$, then

$$
\begin{aligned}
|Q|^{\frac{1}{u}-\frac{1}{p}}\left(\sum_{k: Q_{0, k} \subset Q}\left|\lambda_{k}\right|^{p}\right)^{\frac{1}{p}} \leq & 2^{d\left(\frac{1}{u}-\frac{1}{p}\right)}|\widetilde{Q}|^{\frac{1}{u}-\frac{1}{p}}\left(\sum_{k: Q_{0, k} \subset \widetilde{Q}}\left|(\lambda \mid \widetilde{Q})_{k}\right|^{p}\right)^{\frac{1}{p}} \\
& +|Q|^{\frac{1}{u}-\frac{1}{p}}\left(\sum_{k: Q_{0, k} \subset Q}\left|\lambda_{k}-(\lambda \mid \widetilde{Q})_{k}\right|^{p}\right)^{\frac{1}{p}} \\
\leq & \left(2^{d\left(\frac{1}{u}-\frac{1}{p}\right)}+\varepsilon\right)\left\|\lambda\left|m_{u, p}\|<\| \lambda\right| m_{u, p}\right\|
\end{aligned}
$$

by the choice of $\varepsilon$. Therefore,

$$
\left\|\left.\lambda\left|m_{u, p} \|=\max _{Q \subset \widetilde{Q}}\right| Q\right|^{\frac{1}{u}-\frac{1}{p}}\left(\sum_{k: Q_{0, k} \subset Q}\left|\lambda_{k}\right|^{p}\right)^{\frac{1}{p}}\right.
$$

and the lemma is proved.

Proposition 4.9 Let $1 \leq p<u<\infty$. The dual space to $m_{u, p}^{00}\left(\mathbb{Z}^{d}\right)$ is isometrically isomorphic to $\mathcal{X}_{u, p}\left(\mathbb{Z}^{d}\right)$.

Proof By Proposition 4.5, the space $\mathcal{X}_{u, p}$ is a pre-dual space of $m_{u, p}$. So it is sufficient to show that any functional on $m_{u, p}^{00}$ can be represented by some element of $\mathcal{X}_{u, p}$ with the equality of norms.

First we prove that the space $m_{u, p}^{00}$ can be isometrically embedded into a closed subspace of an appropriate vector valued $c_{0}$ space. Let for $j \in \mathbb{N}_{0}$ and $m \in \mathbb{Z}^{d}$, $A_{j, m}=\ell_{p}\left(Q_{-j, m}, w_{j, m}\right)$ be a weighted finite-dimensional $\ell_{p}$ space, equipped with the norm

$$
\left\|\gamma \mid A_{j, m}\right\|=\left\|\gamma_{\nu}\right\|_{p}^{(j, m)}=\left(\sum_{\nu=1}^{2^{j d}}\left|\gamma_{\nu}\right|^{p}\left|Q_{-j, m}\right|^{\frac{p}{u}-1}\right)^{\frac{1}{p}}=2^{j d\left(\frac{1}{u}-\frac{1}{p}\right)}\left(\sum_{\nu=1}^{2^{j d}}\left|\gamma_{\nu}\right|^{p}\right)^{\frac{1}{p}},
$$

where $\gamma=\left\{\gamma_{v}\right\}_{\nu=1}^{2^{j d}}$.

The space $c_{0}\left(A_{j, m}\right)$ is the space of all sequences $a=\left\{a^{(j, m)}\right\}_{j \in \mathbb{N}_{0}, m \in \mathbb{Z}^{d}}$ with $a^{(j, m)} \in A_{j, m}, j \in \mathbb{N}_{0}, m \in \mathbb{Z}^{d}$, and such that $\left\|a^{(j, m)} \mid A_{j, m}\right\| \rightarrow 0$ if $j+|m| \rightarrow \infty$. We equip $c_{0}\left(A_{j, m}\right)$ with the usual norm, i.e.,

$$
\left\|a\left|c_{0}\left(A_{j, m}\right)\left\|=\sup _{j \in \mathbb{N}_{0}, m \in \mathbb{Z}^{d}}\right\| a^{(j, m)}\right| A_{j, m}\right\| .
$$


It is well known that the dual space of $c_{0}\left(A_{j, m}\right)$ is $\ell_{1}\left(A_{j, m}^{\prime}\right)$, where $b=$ $\left\{b^{(j, m)}\right\}_{j \in \mathbb{N}_{0}, m \in \mathbb{Z}^{d}} \in \ell_{1}\left(A_{j, m}^{\prime}\right)$ means

$$
\left\|b\left|\ell_{1}\left(A_{j, m}^{\prime}\right)\left\|=\sum_{j \in \mathbb{N}_{0}, m \in \mathbb{Z}^{d}}\right\| b^{(j, m)}\right| A_{j, m}^{\prime}\right\|<\infty .
$$

Moreover,

$$
(a, b)=\sum_{j \in \mathbb{N}_{0}, m \in \mathbb{Z}^{d}}\left(a_{j, m}, b_{j, m}\right)
$$

cf., e.g., [51, Lemma 1.11.1].

Let $\lambda=\left\{\lambda_{k}\right\}_{k \in \mathbb{Z}^{d}} \in m_{u, p}^{00}$. We define the mapping

$$
T: m_{u, p}^{00} \ni \lambda \mapsto\left\{\lambda^{(j, m)}\right\}_{j \in \mathbb{N}_{0}, m \in \mathbb{Z}^{d}} \in c_{0}\left(A_{j, m}\right)
$$

by putting $\lambda_{k}^{(j, m)}=\lambda_{k}$ if $Q_{0, k} \subset Q_{-j, m}$.

An argument similar to the one used in the proof of Lemma 4.8 shows that $\left\|T(\lambda) \mid A_{j, m}\right\| \rightarrow 0$ if $j \rightarrow \infty$ or/and $|m| \rightarrow \infty$. Furthermore, by construction,

$$
\left\|\left\{\lambda^{(j, m)}\right\}_{j, m}\left|c_{0}\left(A_{j, m}\right)\|=\| \lambda\right| m_{u, p}\right\| .
$$

So we can identify $m_{u, p}^{00}$ with a closed subspace of $c_{0}\left(A_{j, m}\right)$.

Let $f \in\left(m_{u, p}\right)^{\prime}$. The above identification and the Hahn-Banach theorem imply that $f$ can be extended to a continuous linear functional $\widetilde{f}$ on $c_{0}\left(A_{j, m}\right)$ and $\|\tilde{f}\|=\|f\|$. But $\tilde{f}$ has a representation of the form

$$
\widetilde{f}\left(\left\{\lambda^{(j, m)}\right\}_{j, m}\right)=\sum_{j \in \mathbb{N}_{0}, m \in \mathbb{Z}^{d}} \sum_{k: Q_{0, k} \subset Q_{-j, m}} \lambda_{k}^{(j, m)} \mu_{k}^{(j, m)}
$$

and

$$
\|\tilde{f}\|=\sum_{j \in \mathbb{N}_{0}, m \in \mathbb{Z}^{d}}\left|Q_{-j, m}\right|^{\frac{1}{p}-\frac{1}{u}}\left(\sum_{k: Q_{0, k} \subset Q_{-j, m}}\left|\mu_{k}^{(j, m)}\right|^{p^{\prime}}\right)^{\frac{1}{p^{\prime}}} .
$$

If $\left\{\lambda^{(j, m)}\right\}_{j, m}=T(\lambda)$, then the sum (4.10) can be rearranged in the following way:

$$
f(\lambda)=\tilde{f}(T(\lambda))=\sum_{j=0}^{\infty} \sum_{k \in \mathbb{Z}^{d}} \lambda_{k} \mu_{k}^{(j)}
$$

where the sequence $\mu^{(j)}=\left\{\mu_{k}^{(j)}\right\}_{k \in \mathbb{Z}^{d}}$ is given by $\mu_{k}^{(j)}=\mu_{k}^{(j, m)}$ if $Q_{0, k} \subset Q_{-j, m}$. Recall that for any $k$, there is exactly one cube $Q_{-j, m}$ of size $2^{j d}$ such that $Q_{0, k} \subset$ $Q_{-j, m}$. Moreover, (4.11) reads as 
$\|f\|=\|\tilde{f}\|=\sum_{j=0}^{\infty} 2^{j d\left(\frac{1}{p}-\frac{1}{u}\right)} \sum_{m \in \mathbb{Z}^{d}}\left(\sum_{k: Q_{0, k} \subset Q_{-j, m}} 2^{d j\left(\frac{1}{p}-\frac{1}{u}\right)}\left|\mu_{k}^{(j)}\right|^{p^{\prime}}\right)^{\frac{1}{p^{\prime}}}=\sum_{j=0}^{\infty}\left\|\mu^{(j)}\right\|_{u, p}^{(j)}$

by (4.1). Hence definition (4.6) yields that the sequence $\mu=\sum_{j=0}^{\infty} \mu^{(j)} \in \mathcal{X}_{u, p}$ and

$$
f(\lambda)=\sum_{k \in \mathbb{Z}^{d}}^{\infty} \lambda_{k} \mu_{k} \quad \text { with } \quad\|f\|=\left\|\mu \mid \mathcal{X}_{u, p}\right\| .
$$

Remark 4.10 Similar calculations for the Morrey function spaces can be found in [40] and [29]. In the last paper, Köthe dual spaces to Morrey-type spaces generated by a basis of measurable functions are studied. In particular, Theorems 2.1 and 2.2 ibidem are related to our Propositions 3.5 and 3.9.

Moreover, arguments similar to those used in the proof of Proposition 4.9 show that

$$
\ell_{u}\left(\mathbb{Z}^{d}\right) \hookrightarrow m_{u, p}^{00}\left(\mathbb{Z}^{d}\right) \hookrightarrow c_{0}\left(\mathbb{Z}^{d}\right)
$$

We recall that $m_{u, p}\left(\mathbb{Z}^{d}\right) \hookrightarrow \ell_{\infty}\left(\mathbb{Z}^{d}\right)$, cf. Proposition 2.3 .

\section{Pitt's Compactness Theorem}

Now we prove Pitt's theorem for the Morrey sequence spaces. We follow the approach presented in [9] and [15]. The original result reads as follows.

Theorem 5.1 ([37]) Let $1 \leq q<p<\infty$. Every bounded linear operator from $\ell_{p}$ into $\ell_{q}$ or from $c_{0}$ into $\ell_{q}$ is compact.

We start with the following lemma that shows the similarity of $m_{u, p}^{00}\left(\mathbb{Z}^{d}\right)$ to $c_{0}$ if $p<u$.

Lemma 5.2 Let $0<p<u<\infty$ and $w^{(n)}$ be a sequence in $m_{u, p}^{00}\left(\mathbb{Z}^{d}\right)$, which is weakly convergent to zero, $w_{n} \rightarrow 0$. Then for any $\lambda \in m_{u, p}^{00}\left(\mathbb{Z}^{d}\right)$,

$$
\limsup _{n \rightarrow \infty}\left\|\lambda+w^{(n)} \mid m_{u, p}\left(\mathbb{Z}^{d}\right)\right\|=\max \left\{\left\|\lambda\left|m_{u, p}\left(\mathbb{Z}^{d}\right)\left\|, \limsup _{n \rightarrow \infty}\right\| w^{(n)}\right| m_{u, p}\left(\mathbb{Z}^{d}\right)\right\|\right\} .
$$

Proof Step 1. First we assume that the sequence $\lambda \in m_{u, p}^{00}$ is finite. The sequences $\lambda+w^{(n)}$ and $w^{(n)}$ belong to $m_{u, p}^{00}$, therefore, according to Lemma 4.8, there exist dyadic cubes $Q_{n}$ and $\widetilde{Q}_{n}$ such that 


$$
\begin{gathered}
\left\|\lambda+\left.w^{(n)}\left|m_{u, p} \|=\right| Q_{n}\right|^{\frac{1}{u}-\frac{1}{p}}\left(\sum_{k: Q_{0, k} \subset Q_{n}}\left|\lambda_{k}+w_{k}^{(n)}\right|^{p}\right)^{\frac{1}{p}},\right. \\
\left\|\left.w^{(n)}\left|m_{u, p} \|=\right| \widetilde{Q}_{n}\right|^{\frac{1}{u}-\frac{1}{p}}\left(\sum_{k: Q_{0, k} \subset \widetilde{Q}_{n}}\left|w_{k}^{(n)}\right|^{p}\right)^{\frac{1}{p}} .\right.
\end{gathered}
$$

By the definition of lim sup there is always a subsequence of cubes $\left\{Q_{n_{i}}\right\}_{i}$ such that

$$
\limsup _{n \rightarrow \infty}\left\|\lambda+\left.w^{(n)}\left|m_{u, p} \|=\lim _{i \rightarrow \infty}\right| Q_{n_{i}}\right|^{\frac{1}{u}-\frac{1}{p}}\left(\sum_{k: Q_{0, k} \subset Q_{n_{i}}}\left|\lambda_{k}+w_{k}^{\left(n_{i}\right)}\right|^{p}\right)^{\frac{1}{p}} .\right.
$$

The cubes $Q_{n}$ are dyadic cubes of size at least one; therefore we may assume that the subsequence satisfies one of the following alternative conditions:

(1) there exists a dyadic cube $Q$ such that $Q_{n_{i}} \subset Q$ for any $i$,

(2) $\lim _{i \rightarrow \infty}\left|Q_{n_{i}}\right|=\infty$,

(3) $\sup _{i}\left|Q_{n_{i}}\right|<\infty$ and $Q_{n_{i}} \cap Q_{n_{j}}=\emptyset$ if $i \neq j$.

A similar statement holds for the cubes $\widetilde{Q}_{n}$.

Please note that the weak convergence of the sequence $w^{(n)}$ to zero implies the uniform convergence to zero of the coordinates of $w^{(n)}$ on any dyadic cube $Q$. In the next steps we shall denote by $\widetilde{Q}(\lambda)$ the dyadic cube that contains the support of $\lambda$.

Substep 1.1 We prove that

$$
\max \left\{\left\|\lambda\left|m_{u, p}\left\|, \limsup _{n \rightarrow \infty}\right\| w^{(n)}\right| m_{u, p}\right\|\right\} \leq \limsup _{n \rightarrow \infty}\left\|\lambda+w^{(n)} \mid m_{u, p}\right\| .
$$

We have that

$$
\begin{aligned}
\left\|\lambda \mid m_{u, p}\right\| & =|Q(\lambda)|^{\frac{1}{u}-\frac{1}{p}}\left(\sum_{k: Q_{0, k} \subset Q(\lambda)}\left|\lambda_{k}\right|^{p}\right)^{\frac{1}{p}} \\
& =\lim _{i \rightarrow \infty}|Q(\lambda)|^{\frac{1}{u}-\frac{1}{p}}\left(\sum_{k: Q_{0, k} \subset Q(\lambda)}\left|\lambda_{k}+w_{k}^{\left(n_{i}\right)}\right|^{p}\right)^{\frac{1}{p}} \\
& \leq \lim _{i \rightarrow \infty}\left|Q_{n_{i}}\right|^{\frac{1}{u}-\frac{1}{p}}\left(\sum_{k: Q_{0, k} \subset Q_{n_{i}}}\left|\lambda_{k}+w_{k}^{\left(n_{i}\right)}\right|^{p}\right)^{\frac{1}{p}} \\
& =\limsup _{n \rightarrow \infty}\left\|\lambda+w^{(n)} \mid m_{u, p}\right\|,
\end{aligned}
$$


where we used (5.1). Let

$$
\limsup _{n \rightarrow \infty}\left\|\left.w^{(n)}\left|m_{u, p} \|=\lim _{i \rightarrow \infty}\right| \widetilde{Q}_{n_{i}}\right|^{\frac{1}{u}-\frac{1}{p}}\left(\sum_{k: Q_{0, k} \subset \widetilde{Q}_{n_{i}}}\left|w_{k}^{\left(n_{i}\right)}\right|^{p}\right)^{\frac{1}{p}} .\right.
$$

If the sequence of cubes $\widetilde{Q}_{n_{i}}$ satisfies the condition (5.2), then $\left\|w^{\left(n_{i}\right)} \mid m_{u, p}\right\| \rightarrow 0$. So the inequality (5.5) holds.

If the sequence of cubes $\widetilde{Q}_{n_{i}}$ satisfies the condition (5.3), then

$$
\begin{aligned}
\left|\widetilde{Q}_{n_{i}}\right|^{\frac{1}{u}-\frac{1}{p}} & \left(\sum_{k: Q_{0, k} \subset \widetilde{Q}_{n_{i}}}\left|w_{k}^{\left(n_{i}\right)}\right|^{p}\right)^{\frac{1}{p}} \\
& \leq\left|\widetilde{Q}_{n_{i}}\right|^{\frac{1}{u}-\frac{1}{p}}\left(\sum_{k: Q_{0, k} \subset \widetilde{Q}_{n_{i}}}\left|\lambda_{k}+w_{k}^{\left(n_{i}\right)}\right|^{p}\right)^{\frac{1}{p}}+\left(\frac{\left|\widetilde{Q}_{n_{i}}\right|}{|\widetilde{Q}(\lambda)|}\right)^{\frac{1}{u}-\frac{1}{p}}\left\|\lambda \mid m_{u, p}\right\| .
\end{aligned}
$$

But $\left(\left|\widetilde{Q}_{n_{i}}\right| /|\widetilde{Q}(\lambda)|\right)^{\frac{1}{u}-\frac{1}{p}} \rightarrow \underset{\sim}{0}$, so the inequality (5.5) holds also in this case.

If the sequence of cubes $\widetilde{Q}_{n_{i}}$ satisfies the condition (5.4), then for sufficiently large $i$ we have $\lambda \mid \widetilde{Q}_{n_{i}}=0$, and again the inequality (5.5) holds.

Substep 1.2. Now we prove the inequality converse to (5.5), i.e.,

$$
\limsup _{n \rightarrow \infty}\left\|\lambda+w^{(n)} \mid m_{u, p}\right\| \leq \max \left\{\left\|\lambda\left|m_{u, p}\left\|, \limsup _{n \rightarrow \infty}\right\| w^{(n)}\right| m_{u, p}\right\|\right\} .
$$

We can proceed in a similar way as in the last step, now using the cubes $Q_{n_{i}}$. If the sequence of cubes $Q_{n_{i}}$ satisfies the condition (5.2), then

$$
\left|Q_{n_{i}}\right|^{\frac{1}{u}-\frac{1}{p}}\left(\sum_{k: Q_{0, k} \subset Q_{n_{i}}}\left|w_{k}^{\left(n_{i}\right)}\right|^{p}\right)^{\frac{1}{p}} \rightarrow 0 .
$$

So

$$
\limsup _{n \rightarrow \infty}\left\|\lambda+w^{(n)}\left|m_{u, p}\|\leq\| \lambda\right| m_{u, p}\right\| .
$$

If the sequence of cubes $Q_{n_{i}}$ satisfies the condition (5.3), then similarly as above we conclude

$$
\begin{aligned}
\left|Q_{n_{i}}\right|^{\frac{1}{u}}-\frac{1}{p} & \left(\sum_{k: Q_{0, k} \subset Q_{n_{i}}}\left|\lambda_{k}+w_{k}^{\left(n_{i}\right)}\right|^{p}\right)^{\frac{1}{p}} \\
& \leq\left|Q_{n_{i}}\right|^{\frac{1}{u}-\frac{1}{p}}\left(\sum_{k: Q_{0, k} \subset Q_{n_{i}}}\left|w_{k}^{\left(n_{i}\right)}\right|^{p}\right)^{\frac{1}{p}}+\left(\frac{\left|Q_{n_{i}}\right|}{|\widetilde{Q}(\lambda)|}\right)^{\frac{1}{u}-\frac{1}{p}}\left\|\lambda \mid m_{u, p}\right\|,
\end{aligned}
$$

and the last summand tends to zero when $i \rightarrow \infty$. 
If the sequence of cubes $Q_{n_{i}}$ satisfies the condition (5.4), then once more the sequences $\lambda+w_{k}^{\left(n_{i}\right)}$ and $w_{k}^{\left(n_{i}\right)}$ coincide for sufficiently large $i$.

Step 2. The general case is true by the density of finitely supported sequences in $m_{u, p}^{00}$ since the norm is a Lipschitzian function.

Now we can finally establish Pitt's theorem in our context.

Theorem 5.3 Let $1<p<u<\infty$ and $1 \leq q<\infty$. Then any bounded linear operator $T$ from $m_{u, p}^{00}\left(\mathbb{Z}^{d}\right)$ into $\ell_{q}\left(\mathbb{Z}^{d}\right)$ is compact.

Proof Due to Proposition 4.4, Lemma 4.8, Proposition 4.9, and Lemma 5.2, we can follow the arguments presented in [9]. We only sketch the proof for the convenience of the reader.

The dual space to $m_{u, p}^{00}$ is separable, cf. Proposition 4.4(ii) and Proposition 4.9, so every bounded sequence in $m_{u, p}^{00}$ has a weak Cauchy subsequence and $T$ is compact if it is weak-to-norm continuous.

We may assume that $\|T\|=1$. Let $0<\varepsilon<1$. We choose $x_{\varepsilon} \in m_{u, p}^{00}$ such that $\left\|x_{\varepsilon} \mid m_{u, p}\right\|=1$ and $1-\varepsilon \leq\left\|T\left(x_{\varepsilon}\right) \mid \ell_{q}\right\| \leq 1$. Let $w_{n} \rightarrow 0$ in $m_{u, p}^{00}$, and let $\left\|w^{(n)} \mid m_{u, p}\right\| \leq M$. Lemma 5.2 and the analogous statement for $\ell_{q}$, cf. [9], imply for $t>0$ that

$$
\begin{aligned}
\left\|T\left(x_{\varepsilon}\right) \mid \ell_{q}\right\|^{q}+ & t^{q} \limsup _{n \rightarrow \infty}\left\|T\left(w^{(n)}\right) \mid \ell_{q}\right\|^{q} \\
& =\limsup _{n \rightarrow \infty}\left\|T\left(x_{\varepsilon}+t w^{(n)}\right) \mid \ell_{q}\right\|^{q} \\
& \leq \limsup _{n \rightarrow \infty}\left\|x_{\varepsilon}+t w^{(n)} \mid m_{u, p}\right\|^{q} \\
& =\max \left(\left\|x_{\varepsilon}\left|m_{u, p}\left\|^{q}, t^{q} \limsup _{n \rightarrow \infty}\right\|\left(w^{(n)}\right)\right| m_{u, p}\right\|^{q}\right) .
\end{aligned}
$$

This leads to

$$
\limsup _{n \rightarrow \infty}\left\|T\left(w^{(n)}\right) \mid \ell_{q}\right\|^{q} \leq t^{-q}\left[\max \left(1, t^{q} M^{q}\right)-(1-\varepsilon)^{q}\right] .
$$

The choice $0<\varepsilon \leq \min \left(1, M^{-2 q}\right)$ and $t=\varepsilon^{\frac{1}{2 q}}$ implies

$$
\limsup _{n \rightarrow \infty}\left\|T\left(w^{(n)}\right) \mid \ell_{q}\right\|^{q} \leq \varepsilon^{-1 / 2}\left(1-(1-\varepsilon)^{q}\right) .
$$

Taking the limit with $\varepsilon \rightarrow 0$, we have shown that $\left\|T\left(w^{(n)}\right) \mid \ell_{q}\right\| \rightarrow 0$.

Remark 5.4 One can find in the literature results that can be considered as a quantitative version of Pitt's theorem. For example, one can characterize the compactness of the diagonal operator $D_{\sigma}: \ell_{p} \rightarrow \ell_{q}, 1 \leq q<p<\infty$, in terms of entropy numbers or some $s$-numbers, cf. [7] or [24] and the references given there. Such estimates are not the subject of our paper. In the next section, we comment a bit on the behavior of the entropy numbers of embeddings in the finite dimensional case. 


\section{Finite Dimensional Morrey Sequence Spaces}

Finally we shall briefly deal with finite dimensional sequence spaces related to $m_{u, p}$. We have at least two reasons for doing so. First, in view of Theorem 3.1, there is never a compact embedding between two sequence spaces of Morrey type-whereas any continuous embedding between finite-dimensional spaces is compact. Secondly, when dealing with smoothness Morrey spaces like $\mathcal{N}_{u, p, q}^{s}$ or $\mathcal{E}_{u, p, q}^{s}$, for instance, wavelet decompositions usually lead to appropriate sequence spaces that should be studied in further detail. In this spirit it is quite natural and helpful to understand finite sequence spaces of Morrey type better than so far.

For the latter reason we do not consider finite Morrey sequence spaces as general as possible, but only a special 'level' version of it.

Definition 6.1 Let $0<p \leq u<\infty, j \in \mathbb{N}_{0}$, be fixed and $\mathcal{K}_{j}=\left\{k: Q_{0, k} \subset Q_{-j, 0}\right\}$. We define

$$
\begin{aligned}
& m_{u, p}^{2^{j d}}=\left\{\lambda=\left\{\lambda_{k}\right\}_{k \in \mathcal{K}_{j}} \subset \mathbb{C}:\right. \\
&\left\|\left.\lambda\left|m_{u, p}^{2^{j d}} \|=\sup _{Q_{-v, m} \subset Q_{-j, 0}}\right| Q_{-v, m}\right|^{\frac{1}{u}-\frac{1}{p}}\left(\sum_{k: Q_{0, k} \subset Q_{-v, m}}\left|\lambda_{k}\right|^{p}\right)^{\frac{1}{p}}<\infty\right\},
\end{aligned}
$$

where the supremum is taken over all $\nu \in \mathbb{N}_{0}$ and $m \in \mathbb{Z}^{d}$ such that $Q_{-v, m} \subset Q_{-j, 0}$. Remark 6.2 Similarly one can define spaces related to any cube $Q_{-j, m}, m \in \mathbb{Z}^{d}$, but they are isometrically isomorphic to $m_{u, p}^{2^{j d}}$, so we restrict our attention to the last space.

Clearly, for $u=p$, this space coincides with the usual $2^{j d}$-dimensional space $\ell_{p}^{2^{j d}}$, that is, $m_{p, p}^{2^{j d}}=\ell_{p}^{2^{j d}}$.

Lemma 6.3 Let $0<p_{1} \leq u_{1}<\infty, 0<p_{2} \leq u_{2}<\infty$ and $j \in \mathbb{N}_{0}$ be given. Then the norm of the compact identity operator

$$
\operatorname{id}_{j}: m_{u_{1}, p_{1}}^{2^{j d}} \hookrightarrow m_{u_{2}, p_{2}}^{2^{j d}}
$$

satisfies

$$
\left\|\operatorname{id}_{j}\right\|= \begin{cases}1 & \text { if } p_{1} \geq p_{2} \text { and } u_{2} \geq u_{1}, \\ 1 & \text { if } p_{1}<p_{2} \text { and } \frac{p_{2}}{u_{2}} \leq \frac{p_{1}}{u_{1}}, \\ 2^{j d\left(\frac{1}{u_{2}}-\frac{1}{u_{1}}\right)} & \text { if } p_{1} \geq p_{2} \text { and } u_{2}<u_{1},\end{cases}
$$

and in the remaining case, there is a constant $c, 0<c \leq 1$, independent of $j$ such that

$$
c 2^{j d\left(\frac{1}{u_{2}}-\frac{p_{1}}{u_{1} p_{2}}\right)} \leq\left\|\operatorname{id}_{j}\right\| \leq 2^{j d\left(\frac{1}{u_{2}}-\frac{p_{1}}{u_{1} p_{2}}\right)} \quad \text { if } p_{1}<p_{2} \quad \text { and } \quad \frac{p_{2}}{u_{2}}>\frac{p_{1}}{u_{1}} \text {. }
$$


Proof In the case of $p_{1} \geq p_{2}$, the upper estimate for $\left\|\mathrm{id}_{j}\right\|$ follows from Hölder's inequality and the corresponding relations between $u_{1}$ and $u_{2}$. The lower estimate in the case of $u_{1} \leq u_{2}$ follows by applying the sequence $\lambda=\left\{\lambda_{k}\right\}_{k}$ with $\lambda_{0}=1$ and $\lambda_{k}=0$ if $k \neq 0$. Otherwise, if $u_{1}>u_{2}$, we can use the sequence $\lambda=\left\{\lambda_{k}\right\}_{k}$ with $\lambda_{k} \equiv 1$ for any $k$.

Let now $p_{1}<p_{2}$ and $\frac{p_{2}}{u_{2}} \leq \frac{p_{1}}{u_{1}}$. If $\left\|\lambda \mid m_{u_{1}, p_{1}}^{2^{j d}}\right\|=1$, then

$$
\sum_{k: Q_{0, k} \subset Q_{-v, m}}\left|\lambda_{k}\right|^{p_{2}} \leq \sum_{k: Q_{0, k} \subset Q_{-v, m}}\left|\lambda_{k}\right|^{p_{1}},
$$

since $\left|\lambda_{k}\right| \leq 1$. So for any $v$ with $0 \leq v \leq j$, we have

$$
\begin{aligned}
2^{\nu d\left(\frac{p_{2}}{u_{2}}-1\right)} \sum_{k: Q_{0, k} \subset Q_{-v, m}}\left|\lambda_{k}\right|^{p_{2}} & \leq 2^{\nu d\left(\frac{p_{2}}{u_{2}}-\frac{p_{1}}{u_{1}}\right)} 2^{\nu d\left(\frac{p_{1}}{u_{1}}-1\right)} \sum_{k: Q_{0, k} \subset Q_{-v, m}}\left|\lambda_{k}\right|^{p_{1}} \\
& \leq 2^{v d\left(\frac{p_{2}}{u_{2}}-\frac{p_{1}}{u_{1}}\right)} \leq 1 .
\end{aligned}
$$

This proves that $\left\|\mathrm{id}_{j}\right\| \leq 1$. The opposite inequality can be proved in the same way as in the first case.

If $p_{1}<p_{2}, \frac{p_{2}}{u_{2}}>\frac{p_{1}}{u_{1}}$, and $\left\|\lambda \mid m_{u_{1}, p_{1}}^{2^{j d}}\right\|=1$, then analogously to the above, we can prove that

$$
2^{\nu d\left(\frac{p_{2}}{u_{2}}-1\right)} \sum_{k: Q_{0, k} \subset Q_{-v, m}}\left|\lambda_{k}\right|^{p_{2}} \leq 2^{\nu d\left(\frac{p_{2}}{u_{2}}-\frac{p_{1}}{u_{1}}\right)} .
$$

So

$$
\left\|\lambda \mid m_{u_{2}, p_{2}}^{2^{j d}}\right\| \leq 2^{d j\left(\frac{1}{u_{2}}-\frac{p_{1}}{u_{1} p_{2}}\right)} .
$$

To prove the opposite inequality, we can use the same argument as in Substep 2.2 of the proof of Theorem 3.1. We have

$$
\begin{aligned}
2^{d j\left(\frac{1}{u_{2}}-\frac{p_{1}}{u_{1} p_{2}}\right)} & =2^{d j\left(\frac{1}{u_{2}}-\frac{1}{p_{2}}\right)} 2^{d j\left(1-\frac{p_{1}}{u_{1}}\right) \frac{1}{p_{2}}} \leq c^{-1} 2^{d j\left(\frac{1}{u_{2}}-\frac{1}{p_{2}}\right)} k_{j}^{\frac{1}{p_{2}}} \\
& =c^{-1}\left\|\lambda^{(j)}\left|m_{u_{2}, p_{2}}^{2^{j d}}\|\leq\| \mathrm{id}_{j}\|\| \lambda^{(j)}\right| m_{u_{1}, p_{1}}^{2^{j d}}\right\| \leq c^{-1}\left\|\mathrm{id}_{j}\right\|
\end{aligned}
$$

cf. (3.4)-(3.6).

Remark 6.4 We suppose that (6.4) is in fact an equality as well, but have no proof yet. In that case (6.3) and (6.4) could be summarized as

$$
\left\|\operatorname{id}_{j}\right\| \sim 2^{j d\left(\frac{1}{u_{2}}-\frac{1}{u_{1}} \min \left(1, \frac{p_{1}}{p_{2}}\right)\right)_{+}} .
$$

Finally we want to characterize the compactness of the embedding id ${ }_{j}$ given by (6.2) in some further detail. We restrict ourselves to the study of entropy numbers here, also for later use. Thus let us briefly recall the concept. 
Definition 6.5 Let $A_{1}$ and $A_{2}$ be two complex (quasi-) Banach spaces, $k \in \mathbb{N}$, and let $T: A_{1} \rightarrow A_{2}$ be a linear and continuous operator from $A_{1}$ into $A_{2}$. The $k$ th (dyadic) entropy number $e_{k}$ of $T$ is the infimum of all numbers $\varepsilon>0$ such that there exist $2^{k-1}$ balls in $A_{2}$ of radius $\varepsilon$ that cover the image $T U_{1}$ of the unit ball $U_{1}=\left\{a \in A_{1}:\left\|a \mid A_{1}\right\| \leq 1\right\}$.

For details and properties of entropy numbers, we refer to [7, 10, 21, 36] (restricted to the case of Banach spaces) and [11] for some extensions to quasi-Banach spaces. Among other features we only want to mention the multiplicativity of entropy numbers: let $A_{1}, A_{2}$, and $A_{3}$ be complex (quasi-) Banach spaces and $T_{1}: A_{1} \longrightarrow A_{2}, T_{2}$ : $A_{2} \longrightarrow A_{3}$ two operators in the sense of Definition 6.5. Then

$$
e_{k_{1}+k_{2}-1}\left(T_{2} \circ T_{1}\right) \leq e_{k_{1}}\left(T_{1}\right) e_{k_{2}}\left(T_{2}\right), \quad k_{1}, k_{2} \in \mathbb{N} .
$$

Note that $\lim _{k \rightarrow \infty} e_{k}(T)=0$ if and only if $T$ is compact, which explains the saying that entropy numbers measure "how compact" an operator acts.

One of the main tools in our arguments will be the characterization of the asymptotic behavior of the entropy numbers of the embedding $\ell_{p_{1}}^{N} \hookrightarrow \ell_{p_{2}}^{N}$. We recall it for convenience. For all $n \in \mathbb{N}$, we have in the case of $0<p_{1} \leq p_{2} \leq \infty$ that

$$
e_{k}\left(\mathrm{id}: \ell_{p_{1}}^{N} \hookrightarrow \ell_{p_{2}}^{N}\right) \sim \begin{cases}1 & \text { if } 1 \leq k \leq \log 2 N, \\ \left(\frac{\log \left(1+\frac{N}{k}\right)}{k}\right)^{\frac{1}{p_{1}}-\frac{1}{p_{2}}} & \text { if } \quad \log 2 N \leq k \leq 2 N, \\ 2^{-\frac{k}{2 N} N^{\frac{1}{p_{2}}}-\frac{1}{p_{1}}} & \text { if } \quad 2 N \leq k\end{cases}
$$

and in case $0<p_{2}<p_{1} \leq \infty$, it holds that

$$
e_{k}\left(\mathrm{id}: \ell_{p_{1}}^{N} \hookrightarrow \ell_{p_{2}}^{N}\right) \sim 2^{-\frac{k}{2 N}} N^{\frac{1}{p_{2}}-\frac{1}{p_{1}}} \quad \text { for all } k \in \mathbb{N}
$$

In the case $1 \leq p_{1}, p_{2} \leq \infty$, this has been proved by Schütt [45]. For $p_{1}<1$ and/or $p_{2}<1$, we refer to Edmunds and Triebel [11] and Triebel [52, 7.2, 7.3] (with a little supplement in [23]).

Corollary 6.6 Let $j \in \mathbb{N}, 0<p_{i} \leq u_{i}<\infty, i=1,2$, and $k \in \mathbb{N}_{0}$ with $k \gtrsim 2^{j d}$. Then

$$
e_{k}\left(\operatorname{id}_{j}: m_{u_{1}, p_{1}}^{2^{j d}} \rightarrow m_{u_{2}, p_{2}}^{2^{j d}}\right) \sim 2^{-k 2^{-j d}} 2^{j d\left(\frac{1}{u_{2}}-\frac{1}{u_{1}}\right)}
$$

Remark 6.7 It will be obvious from the proof below that the assumption for $k$ to be sufficiently large, $k \gtrsim 2^{j d}$, is not needed in all cases. But for simplicity we have stated the result above in that setting only. 
Proof The estimate from above follows from the following commutative diagram and the multiplicativity of entropy numbers:

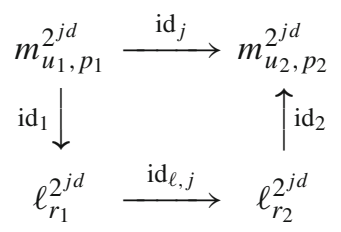

leads to

$$
e_{k}\left(\mathrm{id}_{j}\right) \leq\left\|\operatorname{id}_{1}: m_{u_{1}, p_{1}}^{2^{j d}} \rightarrow \ell_{r_{1}}^{2^{j d}}\right\|\left\|\operatorname{id}_{2}: \ell_{r_{2}}^{2^{j d}} \rightarrow m_{u_{2}, p_{2}}^{2^{j d}}\right\| e_{k}\left(\mathrm{id}_{\ell, j}: \ell_{r_{1}}^{2^{j d}} \rightarrow \ell_{r_{2}}^{2^{j d}}\right) .
$$

We choose $r_{1}=p_{1}$ and $r_{2}=u_{2}$, apply Lemma 6.3, and arrive at

$$
e_{k}\left(\operatorname{id}_{j}\right) \leq 2^{j d\left(\frac{1}{p_{1}}-\frac{1}{u_{1}}\right)} e_{k}\left(\operatorname{id}_{\ell, j}: \ell_{p_{1}}^{2^{j d}} \rightarrow \ell_{u_{2}}^{2^{j d}}\right)
$$

Together with (6.5) and (6.6), this leads to the upper estimate in (6.7), where only in the case of $p_{1} \leq u_{2}$ the additional assumption $k \gtrsim 2^{j d}$ is needed.

Conversely, for the lower estimate we 'reverse' the above diagram, that is, we consider

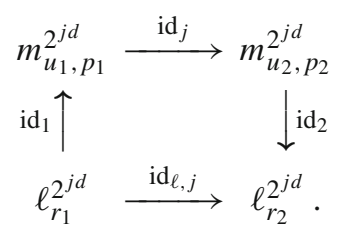

Thus we arrive at

$$
e_{k}\left(\operatorname{id}_{\ell, j}: \ell_{r_{1}}^{2^{j d}} \rightarrow \ell_{r_{2}}^{2^{j d}}\right) \leq\left\|\operatorname{id}_{1}: \ell_{r_{1}}^{2^{j d}} \rightarrow m_{u_{1}, p_{1}}^{2^{j d}}\right\|\left\|\operatorname{id}_{2}: m_{u_{2}, p_{2}}^{2^{j d}} \rightarrow \ell_{r_{2}}^{2^{j d}}\right\| e_{k}\left(\mathrm{id}_{j}\right) .
$$

This time we choose $r_{1}=u_{1}$ and $r_{2}=p_{2}$, apply Lemma 6.3 again, and obtain

$$
e_{k}\left(\operatorname{id}_{\ell, j}: \ell_{u_{1}}^{2^{j d}} \rightarrow \ell_{p_{2}}^{2^{j d}}\right) \leq 2^{j d\left(\frac{1}{p_{2}}-\frac{1}{u_{2}}\right)} e_{k}\left(\mathrm{id}_{j}\right)
$$

Together with (6.5) and (6.6), this completes the argument of the lower estimate in (6.7), where now only in the case of $u_{1} \leq p_{2}$ the additional assumption $k \gtrsim 2^{\text {jd }}$ is needed.

Remark 6.8 It was not our aim here to study $e_{k}\left(\mathrm{id}_{j}\right)$ in all cases, though for several applications also the results (and constants) for small $k \in \mathbb{N}$ are very useful. Moreover, there are further quantities that characterize compactness of operators that admit a lot of further interesting applications. At the moment, we concentrated on the new concept 
of Morrey sequence spaces as introduced in this paper (with some forerunner in [16]) and found in this last section that for sufficiently large $k \in \mathbb{N}, k \gtrsim 2^{j d}$,

$$
e_{k}\left(\mathrm{id}_{j}: m_{u_{1}, p_{1}}^{2^{j d}} \rightarrow m_{u_{2}, p_{2}}^{2^{j d}}\right) \sim e_{k}\left(\mathrm{id}: \ell_{u_{1}}^{2^{j d}} \rightarrow \ell_{u_{2}}^{2^{j d}}\right)
$$

though the corresponding sequence spaces are quite different.

Acknowledgements We are indebted to the referees of the first version of the paper for their valuable remarks that helped to improve the presentation.

Open Access This article is distributed under the terms of the Creative Commons Attribution 4.0 International License (http://creativecommons.org/licenses/by/4.0/), which permits unrestricted use, distribution, and reproduction in any medium, provided you give appropriate credit to the original author(s) and the source, provide a link to the Creative Commons license, and indicate if changes were made.

\section{References}

1. Adams, D.R.: Morrey Spaces. Lecture Notes in Applied and Numerical Harmonic Analysis. Springer, Cham (2015)

2. Adams, D.R., Xiao, J.: Nonlinear potential analysis on Morrey spaces and their capacities. Indiana Univ. Math. J. 53, 1629-1663 (2004)

3. Adams, D.R., Xiao, J.: Morrey potentials and harmonic maps. Commun. Math. Phys. 308, 439-456 (2011)

4. Adams, D.R., Xiao, J.: Morrey spaces in harmonic analysis. Ark. Mat. 50, 201-230 (2012)

5. Alvarez, J.: Continuity of Calderón-Zygmund type operators on the predual of a Morrey space. In: Clifford Algebras in Analysis and Related Topics (Fayetteville, AR, 1993), Studies in Advanced Mathematics, pp. 309-319. CRC, Boca Raton, FL (1996)

6. Berezhnoı̆, E.I.: A discrete version of Morrey local spaces. Izv. Ross. Akad. Nauk Ser. Mat. 81, 3-30 (2017)

7. Carl, B., Stephani, I.: Entropy, Compactness and the Approximation of Operators. Cambridge University Press, Cambridge (1990)

8. Defant, A., López Molina, J.A., Rivera, M.J.: On Pitt's theorem for operators between scalar and vector-valued quasi-Banach sequence spaces. Monatsh. Math. 130, 7-18 (2000)

9. Delpech, S.: A short proof of Pitt's compactness theorem. Proc. AMS 137, 1371-1372 (2009)

10. Edmunds, D.E., Evans, W.D.: Spectral Theory and Differential Operators. Clarendon Press, Oxford (1987)

11. Edmunds, D.E., Triebel, H.: Function Spaces, Entropy Numbers, Differential Operators. Cambridge University Press, Cambridge (1996)

12. El Baraka, A.: An embedding theorem for Campanato spaces. Electron. J. Differ. Equ. 66, 1-17 (2002)

13. El Baraka, A.: Function spaces of BMO and Campanato type. In: Proceedings of the $2002 \mathrm{Fez}$ Conference on Partial Differential Equations, pp. 109-115 (electronic), Electronic Journal of Differential Equations Conference 9, Southwest Texas State University, San Marcos, TX (2002)

14. El Baraka, A.: Littlewood-Paley characterization for Campanato spaces. J. Funct. Spaces Appl. 4, 193-220 (2006)

15. Fabian, M., Zizler, V.: A “nonlinear" proof of Pitt's compactness theorem. Proc. AMS 131, 3693-3694 (2003)

16. Gunawan, H., Kikianty, E., Schwanke, C.: Discrete Morrey spaces and their inclusion properties. Math. Nachr. 291(8-9), 1283-1296 (2018)

17. Gunawan, H., Schwanke, C.: The Hardy-Littlewood maximal operator on discrete Morrey spaces. Mediterr. J. Math. (2019). https://doi.org/10.1007/s00009-018-1277-7

18. Haroske, D.D., Skrzypczak, L.: Continuous embeddings of Besov-Morrey function spaces. Acta Math. Sin. (Engl. Ser.) 28, 1307-1328 (2012)

19. Heil, C.: The Basis Theory Primer. Birkhäuser, Basel (2011)

20. Kalita, E.A.: Dual Morrey spaces. Dokl. Akad. Nauk 361(4), 447-449 (1998) 
21. König, H.: Eigenvalue Distribution of Compact Operators. Birkhäuser, Basel (1986)

22. Kozono, H., Yamazaki, M.: Semilinear heat equations and the Navier-Stokes equation with distributions in new function spaces as initial data. Commun. Partial Differ. Equ. 19, 959-1014 (1994)

23. Kühn, T.: A lower estimate for entropy numbers. J. Approx. Theory 110, 120-124 (2001)

24. Kühn, T.: Entropy numbers in sequence spaces with an application to weighted function spaces. J. Approx. Theory 153, 40-52 (2008)

25. Lemarié-Rieusset, P.G.: The Navier-Stokes equations in the critical Morrey-Campanato space. Rev. Mat. Iberoam. 23, 897-930 (2007)

26. Lemarié-Rieusset, P.G.: The role of Morrey spaces in the study of Navier-Stokes and Euler equations. Eurasian Math. J. 3, 62-93 (2012)

27. Lemarié-Rieusset, P.G.: Multipliers and Morrey spaces. Potential Anal. 38, 741-752 (2013)

28. López Molina, J.A.: Pitt's theorem for operators between general Lorentz sequence spaces. Math. Scan. 90, 101-125 (2002)

29. Mastyło, M., Sawano, Y., Tanaka, H.: Morrey type spaces and its Köthe dual space. Bull. Malays. Math. Sci. Soc. 41, 1181-1198 (2018)

30. Mazzucato, A.: Decomposition of Besov-Morrey spaces. Contemp. Math. 320, 279-294 (2003)

31. Mazzucato, A.: Besov-Morrey spaces: function spaces theory and applications to non-linear PDE. Trans. Am. Math. Soc. 355, 1297-1369 (2003)

32. Morrey, C.B.: On the solutions of quasi-linear elliptic partial differential equations. Trans. Am. Math. Soc. 43, 126-166 (1938)

33. Olsen, P.: Fractional integration, Morrey spaces and Schrödinger equation. Commun. Partial Differ. Equ. 20, 20005-2055 (1995)

34. Piccinini, L.C.: Inclusioni tra spazi di Morrey. Boll. Un. Mat. Ital (4) 2, 95-99 (1969)

35. Piccinini, L.C.: Proprietá di inclusione e interpolazione tra spazi di Morrey e loro generalizzazioni. Ph.D. thesis, Scuola Normale Superiore Pisa (1969)

36. Pietsch, A.: Eigenvalues and $s$-Numbers. Akad. Verlagsgesellschaft Geest \& Portig, Leipzig (1987)

37. Pitt, H.R.: A note on bilinear forms. J. Lond. Math. Soc. 11(1), 174-180 (1932)

38. Rosenthal, M.: Morrey-Räume aus der Sicht der harmonischen Analysis. Master's thesis, FriedrichSchiller-Universität Jena, Germany (2009)

39. Rosenthal, M.: Local means, wavelet bases, representations, and isomorphisms in Besov-Morrey and Triebel-Lizorkin-Morrey spaces. Math. Nachr. 286, 59-87 (2013)

40. Rosenthal, M., Triebel, H.: Morrey spaces, their duals and preduals. Rev. Mat. Complut. 28(1), 1-30 (2015)

41. Sawano, Y.: Wavelet characterizations of Besov-Morrey and Triebel-Lizorkin-Morrey spaces. Funct. Approx. Comment. Math. 38, 93-107 (2008)

42. Sawano, Y.: A note on Besov-Morrey spaces and Triebel-Lizorkin-Morrey spaces. Acta Math. Sin. (Engl. Ser.) 25, 1223-1242 (2009)

43. Sawano, Y., Tanaka, H.: Decompositions of Besov-Morrey spaces and Triebel-Lizorkin-Morrey spaces. Math. Z. 257, 871-905 (2007)

44. Sawano, Y., Tanaka, H.: Besov-Morrey spaces and Triebel-Lizorkin-Morrey spaces for non-doubling measures. Math. Nachr. 282, 1788-1810 (2009)

45. Schütt, C.: Entropy numbers of diagonal operators between symmetric Banach spaces. J. Approx. Theory 40, 121-128 (1984)

46. Sickel, W.: Smoothness spaces related to Morrey spaces—a survey. I. Eurasian Math. J. 3, 110-149 (2012)

47. Sickel, W.: Smoothness spaces related to Morrey spaces-a survey. II. Eurasian Math. J. 4, 82-124 (2013)

48. Sickel, W., Yang, D.C., Yuan, W.: Morrey and Campanato Meet Besov, Lizorkin and Triebel. Springer, Berlin (2010)

49. Tang, L., Xu, J.: Some properties of Morrey type Besov-Triebel spaces. Math. Nachr. 278, 904-917 (2005)

50. Taylor, M.: Analysis on Morrey spaces and applications to Navier-Stokes and other evolution equations. Commun. Partial Differ. Equ. 17, 1407-1456 (1992)

51. Triebel, H.: Interpolation Theory, Function Spaces, Differential Operators. North-Holland, Amsterdam (1978)

52. Triebel, H.: Fractals and Spectra. Birkhäuser, Basel (1997) 
53. Triebel, H.: Local Function Spaces, Heat and Navier-Stokes Equations. EMS Tracts in Mathematics 20. European Mathematical Society (EMS), Zürich (2013)

54. Triebel, H.: Hybrid Function Spaces, Heat and Navier-Stokes Equations. EMS Tracts in Mathematics 24. European Mathematical Society (EMS), Zürich (2015)

55. Yang, D., Yuan, W.: A new class of function spaces connecting Triebel-Lizorkin spaces and Q spaces. J. Funct. Anal. 255, 2760-2809 (2008)

56. Yang, D., Yuan, W.: New Besov-type spaces and Triebel-Lizorkin-type spaces including Q spaces. Math. Z. 265, 451-480 (2010)

57. Yuan, W., Sickel, W., Yang, D.: On the coincidence of certain approaches to smoothness spaces related to Morrey spaces. Math. Nachr. 286, 1571-1584 (2013)

58. Zorko, C.T.: Morrey space. Proc. Am. Math. Soc. 98(4), 586-592 (1986)

Publisher's Note Springer Nature remains neutral with regard to jurisdictional claims in published maps and institutional affiliations. 